Publ. RIMS, Kyoto Univ.

18 (1982), 187-240

\title{
On Energy Inequalities and Regularity of Solutions to Weakly Hyperbolic Cauchy Problems
}

By

\author{
Takeshi MANDAI*
}

\section{Introduction}

In this paper, we study how the regularity of solutions to Cauchy problem for a linear hyperbolic equation is affected by the multiplicity of characteristic roots and by lower order terms of the differential operator.

More precisely, we treat the following Cauchy problem for the operator $P=\sum_{j+|\alpha| \leqq m} a_{j, \alpha}(t, x) D_{t}^{j} D_{x}^{\alpha}$ :

$$
\left\{\begin{array}{l}
P u=f(t, x), \quad T_{-} \leqq t \leqq T_{+}, \\
D_{t}^{j} u_{\mid t=T_{-}}=g_{j}(x) \quad(j=0,1, \cdots, m-1),
\end{array}\right.
$$

where $a_{j, \alpha}$ are $C^{\infty}$-functions and $a_{m, 0} \neq 0$. (Notations and definitions are given later.)

First, we remark the following well-known fact.

Let $P$ be a regularly hyperbolic operator on $\left[T_{-}, T_{+}\right] \times \mathbb{R}^{n}$ (see Definition 1.1 (1)), then for any $f \in C^{\infty}\left(\left[T_{-}, T_{+}\right] \times \mathbb{R}^{n}\right)$ and any $g_{j}$ $\in C^{\infty}\left(\boldsymbol{R}^{n}\right) \quad(j=0,1, \cdots, m-1)$, there exists a unique solution $u(t, x)$ of (0.1) and the following energy inequality holds:

$$
\begin{aligned}
& \sum_{j=0}^{m-1}\left\|D_{t}^{j} u(t, \cdot)\right\|_{s+m-1-j} \leqq C_{s}\left(\int_{T_{-}}^{b}\left\|f\left(t^{\prime}, \cdot\right)\right\|_{s} d t^{\prime}\right. \\
& \left.+\sum_{j=0}^{m-1}\left\|g_{j}\right\|_{s+m-1-j}\right) \quad \text { for } \quad T_{-} \leqq t \leqq T_{+}, s \in \mathbb{R} .
\end{aligned}
$$

Here $C_{s}$ denotes a constant independent of $f, g_{j}(j=0, \cdots, m-1)$.

Communicated by S. Matsuura, March 27, 1981.

* Research Institute for Mathematical Sciences, Kyoto University, Kyoto 606, Japan. 
This inequality and other energy inequalities can be interpreted as representations of the regularity of solutions (cf. the index of well-posedness in [7]).

When we have a multiple characteristic root, (0.2) no longer holds. That is, if we assume the inequality $(0.2)$ for $P$, then we can prove that $P$ is regularly hyperbolic. Further, for some operators the regularity-loss of solutions depends much on lower order terms.

The purpose of this paper is to study, by means of energy inequalities, the difference between the degree of regularity of solutions and that of the Cauchy data, when the operator $P$ has multiple characteristic roots.

In Chapter 1 , we treat the case where $P$ has the constant principal part or $P$ is of constant multiplicity. In these cases the regularity of solutions is exactly determined by the multiplicity. This fact has already been known essentially, but we will give a sketch of the proof in order to establish the results as strong as possible.

In Chapter 2, we treat general operators, and we concentrate our attention on the multiplicity of the characteristic roots. In this case, we can also say that as the multiplicity becomes larger, the regularity of solutions becomes worse. But the regularity is not determined by the multiplicity alone.

Chapter 3 is the most important and interesting chapter in this paper. A phenomenon is known that lower order terms affect the regularity-loss of solutions. (For references, see $\S 3.1$.) We will show that for operators of some types this phenomenon actually occurs.

\section{Notations and Definitions}

We introduce some notations.

$$
\begin{aligned}
& (t, x)=\left(t, x_{1}, \cdots, x_{n}\right),(\tau, \xi)=\left(\tau, \dot{\xi}_{1}, \cdots, \hat{\xi}_{n}\right), \\
& D=\left(D_{t}, D_{x}\right)=\left(D_{t}, D_{x_{1}}, \cdots, D_{x_{n}}\right), \\
& D_{t}=-i \partial_{t}=-i(\partial / \partial t), D_{x_{j}}=-i \partial_{x_{j}}=-i\left(\partial / \partial x_{j}\right) \quad(j=1, \cdots, n) \text { etc. }
\end{aligned}
$$

For $A \subset \boldsymbol{R}^{n+1}, I \subset \boldsymbol{R}$, we write $A_{I}=\{(t, x) \in A ; t \in I\}$ and

$$
\begin{gathered}
C^{\infty}(A)=\{\varphi \text {; there exists an open neighborhood } U \text { of } A \\
\text { and } \left.\widetilde{\varphi} \in C^{\infty}(U) \text { such that }\left.\widetilde{\varphi}\right|_{A}=\varphi\right\},
\end{gathered}
$$




$$
\begin{aligned}
& \mathscr{B}^{\infty}(A)=\left\{\varphi \in C^{\infty}(A) ; \text { any derivative of } \varphi \text { is bounded on } A\right\}, \\
& C_{0}^{\infty}(A)=\left\{\varphi \in C_{0}^{\infty}\left(\boldsymbol{R}^{n+1}\right) ; \operatorname{supp} \varphi \subset A\right\} .
\end{aligned}
$$

$\|v\|_{s}$ denotes the Sobolev norm of order $s \in \mathbb{R}$ on $\mathbb{R}^{n}$, and $\|v\|_{H^{p}(A)}$ denotes the Sobolev norm of order $p$ (an integer) on $A \subset \mathbb{R}^{n+1}$.

Let $P=\sum_{j+|\alpha| \leq m} a_{j, \alpha}(t, x) D_{t}^{j} D_{x}^{\alpha}$ be a partial differential operator on $A$, where $\alpha=\left(\alpha_{1}, \cdots, \alpha_{n}\right) \in \mathbb{N}^{n}(\mathbb{N}=\{0,1,2, \cdots\}),|\alpha|=\sum_{j=1}^{n} \alpha_{j}, D_{x}^{\alpha}=D_{x_{1}}^{\alpha_{1}} \cdots$ $D_{x_{n}}^{\alpha_{n}}$ and $a_{j, \alpha} \in C^{\infty}(A)$, then ord. $P$ denotes the order of $P$ on $A$. Further we write

$$
P_{\mu}(t, x ; \tau, \xi)=\sum_{j+|\alpha|=\mu} a_{j, \alpha}(t, x) \tau^{j} \xi^{\alpha},
$$

where $\xi^{\alpha}=\xi_{1}^{\alpha_{1}} \ldots \xi_{n}^{\alpha_{n}}$, and

$$
P_{(k, \beta)}^{(j, \alpha)}(t, x ; \tau, \xi)=\left(\partial_{t}^{k} \partial_{x}^{\beta} \partial_{\tau}^{j} \partial_{\xi}^{\alpha} P\right)(t, x ; \tau, \xi) .
$$

Definition 0.1 . (1) The roots of $P_{m}(t, x ; \tau, \xi)=0$ as an algebraic equation with respect to $\tau$ is called the charactristic roots of $P$ at $(t, x ; \xi)$, and the maximum of their multiplicities is called the multi. plicity (of the characteristic roots) of $P$ at $(t, x ; \xi)$.

(2) We call $P$ hyperbolic in $A$, if $P_{m}(t, x ; 1,0) \neq 0$ in $A$ and the characteristic roots are all real at any $(t, x ; \xi) \in A \times \mathbb{R}^{n}$.

The term "well-posed" has been used in various meanings. We adopt the following definitions after Ivrii-Petkov [7].

Definition 0.2. Let $V$ be an open domain in $\mathbb{R}^{n+1}$ and $P$ be a differential operator with $C^{\infty}$-coefficients on $V$. We assume $P_{m}(t, x ; 1,0)$ $\neq 0$ in $V$.

(1) We say that the non-characteristic Cauchy problem for $P$ (abbreviated to "the C.P. for $P$ " from now on) is rell-posed in $V_{\left[T_{-}, T_{+}\right]}$ $\left(T_{-}<T_{+}\right)$, if the following two conditions are satisfied.

For any $f \in C_{0}^{\infty}(V)$ which satisfies supp $f \subset V_{\left[T_{-}, \infty\right]}$, there exists $u \in \mathscr{D}^{\prime}(V)^{\dagger}$ such that $\left\{\begin{array}{l}P u=f \quad \text { in } V_{\left(-\infty, T_{.}\right]}, \\ \operatorname{supp} u \subset V_{\left[T_{-}, \infty\right)} .\end{array}\right.$

$\dagger \mathscr{D}^{\prime}(V)$ denotes the space of distributions on $V$. 


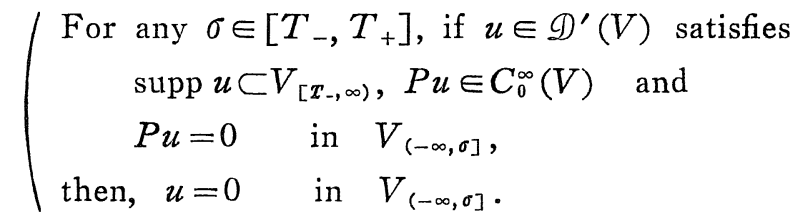

(2) We say that the C.P. for $P$ has a finite propagation speed in $V_{\left[T_{-}, T_{*}\right]}$, if there exists a positive constant $\eta$ such that the following condition is satisfied.

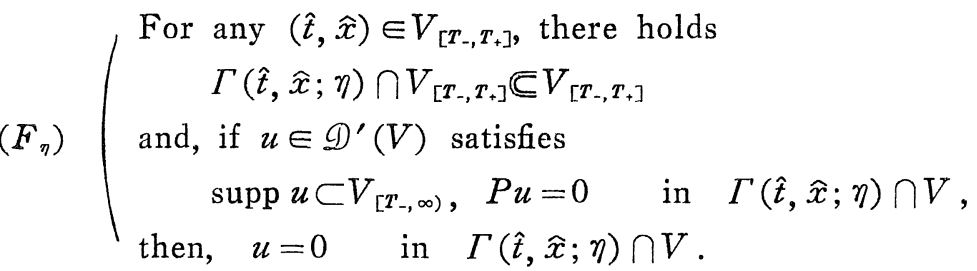

Here, $\Gamma(\hat{t}, \hat{x} ; \eta)=\left\{(t, x) \in \boldsymbol{R}^{n+1} ;|. x-\hat{x}|<\eta|t-\hat{t}|, t<\hat{t}\right\}$, and we write $A \subset B$ if $\bar{A} \subset B$ and $\bar{A}$ is compact.

Now, the next theorem is well-known.

Theorem 0.3. If the C.P. for $P$ is well-posed in $V_{\left[T_{-}, T_{*}\right]}$, then $P$ is hyperbolic in $V_{\left[T_{+}, T_{+}\right]}$. (For the proof in our situation, see [7] or [5].)

Remark 0.4. The proof of the theorem rests only upon the following estimate, which is derived from the assumption of well-posedness.

For any compact set $K \subset V$, there exist a constant $C_{K}$ and integers $p, q$ such that

$$
\begin{aligned}
&\|u\|_{H^{p}\left(V_{\left[T_{-}, t\right]}\right)} \leqq C_{K}\|P u\|_{H^{q}\left(V_{\left[T_{-}, t\right]}\right)} \\
& \text { for } \quad u \in C_{0}^{\infty}\left(K_{\left[T_{-}, T_{+}\right]}\right), \quad t \in\left[T_{-}, T_{+}\right] .
\end{aligned}
$$

\section{Chapter 1. Operators with Constant Principal Part or of Constant Multiplicity}

In this chapter, we treat the operators with constant principal part or of constant multiplicity, and prove that the regularity of solutions is 
exactly determined by the multiplicity of the characteristic roots.

\subsection{The Results}

Definition 1. 1. (1) A partial differential operator $P$ on $A \subset \mathbb{R}^{n+1}$ of order $m$ with $C^{\infty}$-coefficients is called (a hyperbolic operator) of constant multiplicity in $A$, if there exist positive integers $r_{j}(j=1, \cdots, \mu)$ and real-valued functions $\lambda_{j}(t, x ; \xi) \in C^{\infty}\left(A \times\left(\mathbb{R}^{n}-\{0\}\right)\right) \quad(j=1, \cdots, \ell)$ such that

$$
P_{m}(t, x ; \tau, \xi)=\sum_{j=1}^{n}\left(\tau-\lambda_{j}(t, x ; \xi)\right)^{r_{j}} \text { on } A \times \mathbb{R} \times\left(\mathbb{R}^{n}-\{0\}\right),
$$

and

$$
\inf \left\{\left|\lambda_{j}(t, x ; \xi)-\lambda_{k}(t, x ; \xi)\right| ;(t, x) \in A,|\xi|=1, j \neq k\right\}>0 .
$$

When $r_{j}=1 \quad(j=1, \cdots, m)$, we call $P$ regularly hyperbolic in $A$.

(2) Let $P$ be of constant multiplicity in $A$. We say that $P$ satisfies the Levi-Lax condition in $A$, if for any $j$, any open subset $U$ of $A$ and any $\varphi \in C^{\infty}(U)$ which satisfies

$$
\left\{\begin{array}{l}
\partial_{t} \varphi=\lambda_{j}\left(t, x ; \operatorname{grad}_{x} \varphi\right) \\
\operatorname{grad}_{x} \varphi \neq 0
\end{array} \quad \text { on } U\right.
$$

there holds

$$
e^{-i \rho \varphi} P\left(e^{i \rho \varphi} \cdot f\right)=O\left(\rho^{m-r_{j}}\right) \quad(\rho \rightarrow+\infty) \quad \text { for any } f \in C_{0}^{\infty}(U) .
$$

(3) Let $P(\tau, \xi)$ be a polynomial of degree $m$ w.r.t. $(\tau, \xi)$ with constant coefficients. Then $P$ is called a hyperbolic polynomial, if $P_{m}(1,0) \neq 0$ and there exists a constant $C$ such that

$$
P(\tau, \xi) \neq 0 \quad \text { for } \quad \tau \in C,|\operatorname{Im} \tau| \geqq C, \xi \in \mathbb{R}^{n} .
$$

Now, we consider the operator $P$ on $[0, T] \times \mathbb{R}^{n}(T>0)$ with $\mathscr{B}^{\infty}$. coefficients and we assume either of the following two conditions on $P$.

(I) (i) The principal part $P_{m}$ has constant coefficients with $P_{m}(1,0) \neq 0$.

(ii) For any fixed $(\hat{t}, \hat{x}) \in[0, T\rceil \times \mathbb{R}^{n}, P(\hat{t}, \hat{x} ; \tau, \xi)$ is a hyperbolic polynomial in the sense of (3) in Definition 1.1. 
(II) (i) $P$ is a hyperbolic operator of constant multiplicity on $[0, T] \times \boldsymbol{R}^{n}$.

(ii) $P$ satisfies the Levi-Lax condition on $[0, T] \times \boldsymbol{R}^{n}$.

Remark 1.2. In each case, under the condition (i), the condition (ii) is equivalent to that the C.P. for $P$ is well-posed and has a finite propagation speed in $[0, T] \times \boldsymbol{R}^{n}$. (See [7]. [23] and their references.)

The next theorem is the aim of this chapter.

Theorem 1.3. Under the above situation, there hold

(1) Assume that the multiplicity of the characteristic roots at any $(t, x ; \xi) \in[0, T] \times \boldsymbol{R}^{n} \times\left(\boldsymbol{R}^{n}-\{0\}\right)$ is not larger than $r(1 \leqq r \leqq m)$. Then, for any non-negative integer $p$ and any real number $s$, there exists a constant $C_{p, s}$ such that

$$
\begin{aligned}
& \sum_{j=0}^{p+m-r}\left\|D_{t}^{j} u(t, \cdot)\right\|_{s+m-r-j} \\
& \leqq C_{p, s}\left\{\sum_{j=0}^{p} \int_{0}^{t}\left(t-t^{\prime}\right)^{r-1}\left\|D_{t}^{j} P u\left(t^{\prime}, \cdot\right)\right\|_{s-j} d t^{\prime}\right. \\
& +\sum_{j=0}^{p+m-r}\left\|D_{t}^{j} u(0, \cdot)\right\|_{s+m-r-j} \\
& \left.+\sum_{n=1}^{r-1} t^{h} \sum_{j=0}^{p+m-r+h}\left\|D_{t}^{j} u(0, \cdot)\right\|_{s+m-r+h-j}\right\} \\
& \quad \text { for } 0 \leqq t \leqq T, u \in C_{0}^{\infty}\left(\boldsymbol{R}^{n+1}\right) .
\end{aligned}
$$

(2) Let $U$ be an open subset of $[0, T] \times \boldsymbol{R}^{n}$ and $p$, $d$ be integers. If the following inequality holds, then the multiplicity of $P$ is not larger than $d$ at every $(t, x ; \xi) \in[0, T] \times \boldsymbol{R}^{n} \times\left(\boldsymbol{R}^{n}-\{0\}\right)$.

$$
\|u\|_{H^{p+m-d}(U)} \leqq C\|P u\|_{H^{p}(U)} \quad \text { for any } u \in C_{0}^{\infty}(U) .
$$

Here, $C$ is a constant independent of $u$.

Thus, in these cases, the regularity of solutions is exactly determined by the multiplicity of characteristic roots.

The essence of Theorem 1.3 has already been known. But, no inequality as strong as (1.1.1) seems to have been treated so far and 
the proof of (2) has not been stated. So, we will prove this theorem briefly.

\section{$\S 1.2$ Proof of Theorem $\mathbb{1} .3$ (1) in the Case (I)}

First, we review some results from [19].

Definition 1.4. (1) Let $P(\tau, \xi), Q(\tau, \xi)$ be polynomials in $(\tau, \xi)$ with constant coefficients. We write $Q<P$, if there exists a constant $C$ such that

$$
\begin{array}{r}
\sum_{j, \alpha}\left|Q^{(j, \alpha)}(\tau, \xi)\right|^{2} \leqq C \sum_{j, \alpha}\left|P^{(j, \alpha)}(\tau, \xi)\right|^{2} \\
\text { for any }(\tau, \xi) \in \mathbb{R}^{n+1}
\end{array}
$$

(2) When $P_{m}(1,0) \neq 0$, we write $\tau_{1}(\xi), \cdots, \tau_{m}(\xi)$ the characteristic roots of $P(\tau, \xi)$ at $\xi \in \mathbb{R}^{n}$. And, for $k=0,1, \cdots, m$, we put

$$
\begin{aligned}
L_{k}\left(P_{m} ; \tau, \xi\right) & =\sum_{n(J)=k}\left|\frac{P_{m}(\tau, \xi)}{\prod_{j \in J}\left(\tau-\tau_{j}(\xi)\right)}\right|^{2} \\
& =\left|P_{m}(1,0)\right|^{2} \sum_{n(J)=m-k}\left|\prod_{j \in J}\left(\tau-\tau_{j}(\xi)\right)\right|^{2},
\end{aligned}
$$

where $n(J)$ denotes the number of elements of $J \subset\{1, \cdots, m\}$.

Note that $L_{k}$ is a homogeneous polynomial of degree $2(m-k)$ w.r.t. $(\tau, \xi)$.

Proposition 1.5. Let $P(\tau, \xi)$ be a polynomial of degree $m$ w.r.t. $(\tau, \xi)$ with constant coefficients, then the following two conditions are equivalent.

(i) $P$ is a hyperbolic polynomial in the sense of (3) in Definition 1. 1 .

(ii) $P_{m}$ is hyperbolic and $P_{m-k}<P_{m}(k=1, \cdots, m)$. Further, for a homogeneous polynomial $Q(\tau, \xi)$ of degree $m-k(k=1$ $\cdots, m), Q<P_{m}$ if and only if there exists a constant $C$ such that

$$
|Q(\tau, \xi)|^{2} \leqq C L_{k}\left(P_{m} ; \tau, \xi\right) \quad \text { for any } \quad(\tau, \xi) \in \mathbb{R}^{n^{\perp 1}}
$$


The argument in [18] combined with a result of [19] leads to the following proposition.

Proposition 1. 6. There exist $A_{j}^{m-i}(u)(j=0,1, \cdots, n ; i=1, \cdots, m)$ which are real quadratic forms w.r.t. $\left\{D_{t}^{j} D_{x}^{\alpha} u ; j+|\alpha|=m-i\right\}$ such that

$$
\begin{array}{r}
-\operatorname{Im}\left\{P_{m}^{(i-1,0)}\left(D_{t}, D_{x}\right) u \cdot P_{m}^{(i, 0)}\left(D_{t}, D_{x}\right) u\right\} \\
=\partial_{t}\left(A_{0}^{m-i}(u)\right)+\sum_{j=1}^{n} \partial_{x_{j}}\left(A_{j}^{m-i}(u)\right) \\
\text { for any } u \in C^{\infty}\left(\boldsymbol{R}^{n+1}\right) .
\end{array}
$$

Further, if $Q(\tau, \xi)$ is a homogeneous polynomial of degree $m-i$ and $Q<P_{m}$, then there exists a constant $C$ such that

$$
\begin{array}{r}
\left\|Q\left(D_{t}, D_{x}\right) u(t, \cdot)\right\|_{0} \leqq C\left(\int A_{0}^{m-i}(u)(t, x) d x\right)^{1 / 2} \\
\text { for any } t \in \boldsymbol{R}, u \in \mathscr{S}\left(\boldsymbol{R}^{n+1}\right)^{\dagger} .
\end{array}
$$

Now, we sketch the proof of Theorem 1.3 (1) in the case (I). Hereafter, $C$ denotes a constant which may be different in each case.

Integrating (1.2.1) w.r.t. $x$ and using (1.2.2) for $Q=P_{m}^{(i, 0)}$, we have (by $P_{m}^{(i, 0)}<P_{m}$ )

$$
\begin{array}{r}
\partial_{t}\left(\int A_{0}^{m-i}(u)(t, x) d x\right)^{1 / 2} \leqq C\left\|P_{m}^{(i-1,0)}\left(D_{t}, D_{x}\right) u(t, \cdot)\right\|_{0} \\
\text { for any } t \in \boldsymbol{R}, u \in \mathscr{S}\left(\boldsymbol{R}^{n+1}\right)(i=1, \cdots, m) .
\end{array}
$$

Integrating this inequality w.r.t. $t$ and using (1.2.2), we have

$$
\begin{aligned}
& \left\|P_{m}^{(i, 0)}\left(D_{t}, D_{x}\right) u(t, \cdot)\right\|_{0} \leqq C\left(\int A_{0}^{m-i}(u)(t, x) d x\right)^{1 / 2} \\
& \leqq C\left(\int_{0}^{t}\left\|P_{m}^{(i-1,0)}\left(D_{t}, D_{x}\right) u\left(t^{\prime}, \cdot\right)\right\|_{0} d t^{\prime}\right. \\
& \left.\quad+\sum_{j=0}^{m-i}\left\|D_{t}^{j} u(0, \cdot)\right\|_{m-i-j}\right)
\end{aligned}
$$

Successive uses of this inequality leads to

$\dagger \mathscr{S}\left(\boldsymbol{R}^{n+1}\right)$ denotes the Schwartz space on $\boldsymbol{R}^{n+1}$. 
(1. 2.4)

$$
\begin{aligned}
& \left(\int A_{0}^{m-i}(u)(t, x) d x\right)^{1 / 2} \\
& \leqq C\left\{\int_{0}^{t}\left(t-t^{\prime}\right)^{i-1}\left\|P_{m}\left(D_{t}, D_{x}\right) u\left(t^{\prime}, \cdot\right)\right\|_{0} d t^{\prime}\right. \\
& \left.\quad+\sum_{j=0}^{m-i}\left\|D_{t}^{j} u(0, \cdot)\right\|_{m-i-j}+\sum_{h=1}^{i-1} t^{h} \sum_{j=0}^{m-i+h}\left\|D_{t}^{j} u(0, \cdot)\right\|_{m-i+h-j}\right\} .
\end{aligned}
$$

In this inequality, we substitute $D_{t}^{l}\left(1+\left|D_{x}\right|^{2}\right)^{(s-l) / 2} u(l=0,1, \cdots, p)$ for $u$. Then combining the obtained inequality with (1.2.2), we have the following.

If $Q$ is a polynomial of degree $m-i$ with constant coefficients and $Q<P_{m}$, then

$$
\begin{aligned}
& \sum_{j=0}^{p}\left\|D_{t}^{j} Q\left(D_{t}, D_{x}\right) u(t, \cdot)\right\|_{s-j} \\
& \leqq C\left\{\sum_{j=0}^{p} \int_{0}^{t}\left(t-t^{\prime}\right)^{i-1}\left\|D_{t}^{j} P_{m}\left(D_{t}, D_{x}\right) u\left(t^{\prime}, \cdot\right)\right\|_{s-j} d t^{\prime}\right. \\
& \quad+\sum_{j=0}^{p+m-i}\left\|D_{t}^{i} u(0, \cdot)\right\|_{s+m-i-j} \\
& \left.+\sum_{h=1}^{i-1} t^{h} \sum_{j=0}^{p+m-i+h}\left\|D_{t}^{j} u(0, \cdot)\right\|_{s+m-i+h-j}\right\} \\
& \quad \text { for any } \quad t \in[0, T], u \in \mathscr{S}\left(\mathbb{R}^{n+1}\right) \quad(i=1, \cdots, m) .
\end{aligned}
$$

Now, from the assumption (I) and Proposition 1.5, we can write (1.2.6)

$$
\begin{aligned}
P & =P_{m}\left(D_{t}, D_{x}\right)+R\left(t, x ; D_{t}, D_{x}\right) \\
& =P_{m}\left(D_{t}, D_{x}\right)+\sum_{j=1}^{N} b_{j}(t, x) Q_{j}\left(D_{t}, D_{x}\right),
\end{aligned}
$$

where $b_{j} \in \mathscr{B}^{\infty}\left([0, T] \times \mathbb{R}^{n}\right), Q_{j}$ is a polynomial of degree at most $m-1$ with constant coefficients and $Q_{j}<P_{m}$. So, from (1.2.5), we have

(1.2.7) $\quad \sum_{j=0}^{p}\left\|D_{t}^{j} R u(t, \cdot)\right\|_{s-j} \leqq C\left\{\sum_{j=0}^{p} \int_{0}^{t}\left\|D_{t}^{j} P_{m} u\left(t^{\prime}, \cdot\right)\right\|_{s-j} d t^{\prime}\right.$

$$
\left.+\sum_{j=0}^{p+m-1}\left\|D_{t}^{j} u(0, \cdot)\right\|_{s+m-1-j}\right\}
$$

and

$$
\sum_{j=0}^{p}\left\|D_{t}^{j} P_{m} u(t, \cdot)\right\|_{s-j}
$$




$$
\begin{aligned}
\leqq & \sum_{j=0}^{p}\left\|D_{t}^{j} P u(t, \cdot)\right\|_{s-j}+\sum_{j=0}^{p}\left\|D_{t}^{j} R u(t, \cdot)\right\|_{s-j} \\
\leqq & \sum_{j=0}^{p}\left\|D_{t}^{j} P u(t, \cdot)\right\|_{s-j}+C\left\{\sum_{j=0}^{p} \int_{0}^{t}\left\|D_{t}^{j} P_{m} u\left(t^{\prime}, \cdot\right)\right\|_{s-j} d t^{\prime}\right. \\
& \left.+\sum_{j=0}^{p+m-1}\left\|D_{t}^{j} u(0, \cdot)\right\|_{s+m-1-j}\right\} .
\end{aligned}
$$

By Gronwall's lemma (see, for example, [12; Lemma 3]), we have

$$
\begin{aligned}
& \sum_{j=0}^{p} \int_{0}^{t}\left\|D_{t}^{j} P_{m} u\left(t^{\prime}, \cdot\right)\right\|_{s-j} d t^{\prime} \\
& \leqq C\left\{\sum_{j=0}^{p} \int_{0}^{t}\left\|D_{t}^{j} P u\left(t^{\prime}, \cdot\right)\right\|_{s-j} d t^{\prime}+\sum_{j=0}^{p+m-1} t\left\|D_{t}^{j} u(0, \cdot)\right\|_{s+m-1-j}\right\} .
\end{aligned}
$$

By the definitions of $L_{k}$ and $r$, we have

$$
L_{m-k}\left(P_{m} ; \tau, \xi\right)>0 \quad \text { for any } \quad(\tau, \xi) \in \boldsymbol{R}^{n+1}-\{0\}, \quad \text { if } k \leqq m-r .
$$

Further, $L_{m-k}$ is homogeneous of degree $2 k$, so, by Proposition 1.5,

$$
\tau^{j} \xi^{\alpha}<P_{m} \quad \text { if } \quad j \cdot|\alpha| \leqq m-r .
$$

Combining this with $(1.2 .5),(1.2 .8)$, we have the desired result.

Q.E.D.

\section{$\S 1.3$. Proof of Theorem 1.3 (I) in the Case (II)}

Combining the arguments in [3], [10], we have

Proposition 1.7. If $P$ satisfies the condition (II) and has $\mathscr{B}^{\infty}$. coefficients, then there exists regularly hyperbolic operators $R_{k}$ with $\mathscr{B}^{\infty}$-coefficients and partial differential operators $B_{k}$ with $\mathscr{B}^{\infty}$-coeffcients $\left(k=1, \cdots, r=\max _{1 \leqq j \leqq \mu} r_{j}\right)$ such that

$$
\left\{\begin{array}{l}
P=R_{1} \cdots R_{r}+\sum_{k=1}^{r} B_{k} R_{k+1} \cdots R_{r} \quad(\text { as differential operators }) \\
\text { ord. } B_{k} \leqq m_{1}+\cdots+m_{k}-k \quad\left(m_{k}=\text { ord. } R_{k}\right) .
\end{array}\right.
$$

Remark 1.8. (a) Conversely, it can be proved that if $P$ has constant multiplicity, $r=\max _{1 \leqq j \leqq \mu} r_{j}$ and $P$ can be decomposed as above, then $P$ satisfies the Levi-Lax condition.

(b) The Levi-Lax condition in [10] is different from ours. But 
the argument in [3] shows that these are equivalent.

Now, we have only to prove the next theorem.

Theorem 1.9. Let $R_{k}$ be regularly hyperbolic operators on $[0$, $T] \times \mathbb{R}^{n}$ with $\mathscr{B}^{\infty}$-coefficients of order $m_{k}$ and $B_{k}$ be differential operators on $[0, T] \times \mathbb{R}^{n}$ with $\mathscr{B}^{\infty}$-coefficients and ord. $B_{k} \leqq b_{k}=m_{1}+\cdots$ $+m_{k}-k(k=1, \cdots, r)$. If we put

$$
P=R_{1} \cdots R_{r}+\sum_{k=1}^{r} B_{k} R_{k+1} \cdots R_{r},
$$

then the inequality (1.1.1) holds for $P$, where $m=\sum_{k=1}^{r} m_{k}$.

Remark 1.10. The C.P. for $P$ which can be decomposed as above is well-posed and has a finite propagation speed. ([10; Theorem 5.1.])

Proof of Theorem 1.9. We use the following well-known theorem.

Theorem 1.11. Let $R$ be a regularly hyperbolic operator on $[0, T] \times \mathbb{R}^{n}$ with $\mathscr{B}^{\infty}$-coefficients of order $m$. Then, for any non-negative integer $p$ and any real number $s$, there exists a constant $C_{p, s}$ such that

$$
\begin{gathered}
\sum_{j=1}^{p+m-1}\left\|D_{t}^{j} u(t, \cdot)\right\|_{s+m-1-j} \leqq C_{p, s}\left\{\sum_{j=0}^{p} \int_{0}^{t}\left\|D_{t}^{j} P u\left(t^{\prime}, \cdot\right)\right\|_{s-j} d t^{\prime}\right. \\
\left.+\sum_{j=0}^{p+m-1}\left\|D_{t}^{j} u(0, \cdot)\right\|_{s+m-1-j}\right\} \\
\text { for any } 0 \leqq t \leqq T, u \in \mathscr{S}\left(\mathbb{R}^{n+1}\right) .
\end{gathered}
$$

We substitute $R_{k+1} \cdots R_{r} u, R_{k}, p+b_{k-1}, s+b_{k-1}$ and $m_{k}$ for $u, P, p$, $s$ and $m$ in (1.3.1). Then, we have

$$
\begin{aligned}
& \sum_{j=0}^{p+b_{k}}\left\|D_{t}^{j} R_{k+1} \cdots R_{r} u(t, \cdot)\right\|_{s+b_{k}-j} \\
& \leqq C\left\{\sum_{j=0}^{p+b_{k-1}} \int_{0}^{t}\left\|D_{t}^{j} R_{k} \cdots R_{r} u\left(t^{\prime}, \cdot\right)\right\|_{s+b_{k-1}-j} d t^{\prime}\right. \\
&\left.+\sum_{j=0}^{p+m-k}\left\|D_{t}^{j} u(0, \cdot)\right\|_{s+m-k-j}\right\}, \quad(k=1, \cdots, r) .
\end{aligned}
$$


Successive uses of this inequality shows that if we write $\Pi_{m}=R_{1} \cdots R_{r}$ and $R=\sum_{k=1}^{r} B_{k} R_{k+1} \cdots R_{r}$, then

$$
\begin{aligned}
& \sum_{j=1}^{p+m-r}\left\|D_{t}^{j} u(t, \cdot)\right\|_{s+m-r-j} \\
& \leqq C\left\{\sum_{j=0}^{p} \int_{0}^{t}\left(t-t^{\prime}\right)^{r-1}\left\|D_{t}^{j} \Pi_{m} u\left(t^{\prime}, \cdot\right)\right\|_{s-j} d t^{\prime}\right. \\
&+\sum_{j=0}^{p+m-r}\left\|D_{t}^{j} u(0, \cdot)\right\|_{s+m-r-j} \\
&\left.+\sum_{h=1}^{r-1} t^{h} \sum_{j=0}^{p+m-r+h}\left\|D_{t}^{j} u(0, \cdot)\right\|_{s+m-r+h-j}\right\},
\end{aligned}
$$

and

$$
\text { (1.3.3) } \begin{gathered}
\sum_{j=0}^{p}\left\|D_{t}^{j} R u(t, \cdot)\right\|_{s-j} \leqq C\left\{\sum_{j=0}^{p} \int_{0}^{t}\left\|D_{t}^{j} \Pi_{m} u\left(t^{\prime}, \cdot\right)\right\|_{s-j} d t^{\prime}\right. \\
\left.+\sum_{j=0}^{p+m-1}\left\|D_{t}^{j} u(0, \cdot)\right\|_{s+m-1-j}\right\} .
\end{gathered}
$$

(1.3.3) is the same inequality as (1.2.7) in Section 1.2, except that $\Pi_{m}$ replaces $P_{m}$. So, we get (1.2.8) for $\Pi_{m}$ instead of $P_{m}$ as in Section 1.2. Combining this with (1.3.2), we get the desired result.

Q.E.D.

\section{$\S$ 1.4. Proof of Theorem 1.3 (2)}

We need the following lemma in [19].

Lemma 1.12. Let $P$ be a hyperbolic polynomial of degree $m$ with constant coefficients, $r$ be a positive integer and $\hat{\xi} \in \boldsymbol{R}^{n}$. If $\hat{\tau} \in \boldsymbol{R}$ is a root of $P_{m}(\tau, \hat{\xi})=0$ with multiplicity $r$, then

$$
P_{m-k}^{(j, \alpha)}(\hat{\tau}, \hat{\xi})=0 \quad \text { for } \quad j+|\alpha|<r-k, k=0,1, \cdots, r-1 .
$$

Now, we assume that $P$ has a characteristic root $\hat{\tau}$ of multiplicity $r$ at $(\hat{t}, \hat{x} ; \hat{\xi}) \in[0, T] \times \boldsymbol{R}^{n} \times\left(\boldsymbol{R}^{n}-\{0\}\right)$. In the case (I), we put $\varphi(t, x)$ $=\hat{\tau} t+\langle\hat{\xi}, x\rangle$. In the case (II), we take $\varphi$ as the solution of

$$
\left(\begin{array}{l}
\partial_{t} \varphi=\lambda_{j}\left(t, x ; \operatorname{grad}_{x} \varphi\right) \\
\varphi(\hat{t}, x)=\langle\hat{\xi}, x\rangle, \quad \text { where } \quad \lambda_{j}(\hat{t}, \hat{x} ; \hat{\xi})=\hat{\tau} .
\end{array}\right.
$$


This solution can be found in a neighborhood of $(\hat{t}, \hat{x})$ and we can take this neighborhood $\widetilde{U}$ so small that $\partial_{x_{0}} \varphi \neq 0$ on $\widetilde{U}$ for some $j_{0}$. We may assume $\widetilde{U}=U$. And in each case, we put

$$
E_{\rho}(t, x)=e^{i \rho \varphi(t, x)} \quad(\rho \geq 1) .
$$

In the case (I), for any $f \in C_{0}^{\infty}(U)$, by Leibniz' formula,

$$
\begin{aligned}
P_{m-k}\left(f \cdot E_{\rho}\right) & =\sum_{j, \alpha} \frac{1}{j ! \alpha !}\left(D_{t}^{j} D_{x}^{\alpha} f\right) \cdot P_{m-k}^{(j, \alpha)}\left(E_{\rho}\right) \\
& =\sum_{j, \alpha} \frac{1}{j ! \alpha !}\left(D_{t}^{j} D_{x}^{\alpha} f\right) \cdot P_{m-k}^{(j, \alpha)}(t, x ; \hat{\tau}, \hat{\xi}) \rho^{m-k-j-\mid \alpha !} E_{\rho}(t, x) .
\end{aligned}
$$

Here, by the assumption (I) (ii) and Lemma 1.12, we have

$$
P\left(f \cdot E_{\rho}\right)=\sum_{h=0}^{m-r} \rho^{m-r-h} F_{h}(t, x) E_{\rho}(t, x),
$$

where $F_{h} \in C_{0}^{\infty}(U) \quad(h=0,1, \cdots, m-r)$.

In the case (II), by the Levi-Lax condition, we also have (1.4.1).

We will prove that if $f \not \equiv$, for any integers $p, q$, the following estimates hold for sufficiently large $\rho$.

(1) $\left\|f \cdot E_{\rho}\right\|_{H^{p}(U)} \geqq \delta \cdot \rho^{p} \quad(\delta>0)$,

(2) $\left\|P\left(f \cdot E_{\rho}\right)\right\|_{H^{q}(U)} \leqq C \cdot \rho^{m-r+q}$.

If these are proved, from the assumption (1.1.2), we have

$$
p+m-d \leqq m-r+p, \text { that is, } r \leqq d .
$$

Now, when $p \geqq 0, q \geqq 0,(1),(2)$ are almost trivial.

When $p \leqq 0$,

$$
\left\|f \cdot E_{\rho}\right\|_{H^{p}(U)}=\sup _{w \in C_{0}^{\infty}(U)} \frac{\left|\left(f \cdot E_{\rho}, r\right)_{L^{2}(U)}\right|}{\|w\|_{H^{-p}(U)}} .
$$

Here, we will take $w=\widetilde{w}(t, x) E_{\rho}(t, x) \quad\left(\widetilde{w} \in C_{0}{ }^{\infty}(U)\right)$, then

$$
\|w\|_{H^{-p}(U)} \leqq \rho^{-p}\|\widetilde{w}\|_{H^{-p}(U)} .
$$

So,

$$
\left\|f \cdot E_{\rho}\right\|_{H^{p}(U)} \geqq \sup _{\widetilde{w} \in C_{0}^{\infty}(U)} \frac{\left|(f, \widetilde{\widetilde{w}})_{L^{2}(U)}\right|}{\|\widetilde{\widetilde{w}}\|_{H^{-p}(U)}} \cdot \rho^{p}=\rho^{p}\|f\|_{H^{p}(U)} .
$$

When $q \leqq 0$, we take $j_{0}$ such that $\partial_{x_{j_{0}}} \varphi \neq 0$ on $U$, and we solve the 
following equation asymptotically:

$$
D_{x_{j_{0}}}^{|q|} W=P\left(f \cdot E_{\rho}\right)=\rho^{m-r} \sum_{h=0}^{m-r} \rho^{-h} F_{h} E_{\rho} .
$$

We can write for $\psi \in C_{0}^{\infty}(U)$,

$$
D_{x_{j}}^{|q|}\left(\psi \cdot E_{\rho}\right)=E_{\rho} \rho^{|q|} \sum_{h=0}^{|q|} \rho^{-h} \Phi_{h}(\psi),
$$

where $\Phi_{h}$ is a differential operator of order $h$ and

$$
\Phi_{0}=\left(\partial_{x_{j 0}} \varphi\right)^{|q|} \text {. }
$$

So, if we put

$$
W=\rho^{m-r-|q|} \sum_{j=0}^{N} \rho^{-j} w_{j} E_{\rho},
$$

we have

$$
D_{x_{j}}^{|q|} W=\rho^{m-r} \sum_{j=0}^{N+|q|} \rho^{-j} \sum_{k=0}^{|q|} \Phi_{k}\left(w_{j-k}\right) \cdot E_{\rho}
$$

(we take $w_{-1}=w_{-2}=\cdots=w_{-|q|}=0$ ).

If we take $w_{j}$ as

$$
\begin{gathered}
\left(\partial_{x_{j}} \phi\right)^{|q|} w_{j}=F_{j}-\sum_{k=1}^{\mid q i} \Phi_{k}\left(w_{j-k}\right) \quad(j=0,1, \cdots, N) \\
\left(F_{j}=0 \quad \text { for } \quad j \geqq m-r+1\right),
\end{gathered}
$$

then $w_{j} \in C_{0}^{\infty}(U) \quad(j=0,1, \cdots, N)$, and

$$
D_{x_{j_{0}}}^{|q|} W-P\left(f \cdot E_{\rho}\right)=\rho^{m-r-N-1} E_{\rho} \sum_{j=0}^{|q|-1} \rho^{-j} R_{j},
$$

where $R_{j} \in C_{0}{ }^{\infty}(U)$. Now,

$$
\begin{aligned}
\left\|P\left(f \cdot E_{\rho}\right)\right\|_{H^{q}(U)} & \leqq\left\|D_{x_{j_{0}}}^{|q|} W\right\|_{H^{q}(U)}+\left\|D_{x_{j_{0}}}^{|q|} W-P\left(f \cdot E_{\rho}\right)\right\|_{L^{2}(U)} \\
& \leqq \sup _{w \in C_{0}^{\infty}(U)} \frac{\left|\left(D_{x_{0}}^{|q|} W, w\right)_{L^{2}(U)}\right|}{\left\|D_{x_{j_{0}}}^{|q|} w\right\|_{L^{2}(U)}}+C \cdot \rho^{m-r-N-1} \\
& \leqq \sup _{w \in C_{0}^{\infty}(U)} \frac{\left|(W, w)_{L^{2}(U)}\right|}{\|w\|_{L^{2}(U)}}+C \cdot \rho^{m-r-N-1} \\
& =\|W\|_{L^{2}(U)}+C \cdot \rho^{m-r-N-1} \\
& \leqq C \cdot \rho^{m-r-|q|}
\end{aligned}
$$

by taking $N$ sufficiently large.

Q.E.D. 
Chapter 2. The Relation between the Multiplicity of the Characteristic Roots and the Order of Differentiation in Energy Inequality for General Hyperbolic Operators

In the cases treated in Chapter 1 , the regularity of solutions are exactly determined by the multiplicity of characteristic roots. But in general cases, this no longer holds. Typical examples are given in Chapter 3. In this chapter, we study what can be said of the multiplicity of characteristic roots from energy inequalities in general cases.

We consider general operators of the following form on $V_{[0, T]}$.

$$
P=\sum_{j+|\alpha| \leqq m} a_{j, \alpha}(t, x) D_{t}^{j} D_{x}^{\alpha},
$$

where $a_{j, \alpha} \in \mathscr{B}^{\infty}\left(V_{[0, T]}\right), a_{m, 0} \equiv 1, V$ is an open neighborhood of the origin in $\mathbb{R}^{n+1}$.

\section{§ 2.1. The Results}

The next fact stated in Introduction is well-known.

Theorem 2.1. If we assume

$$
\begin{aligned}
& \sum_{j=0}^{m-1}\left\|D_{t}^{j} u(t, \cdot)\right\|_{m-1-j} \leqq C \int_{0}^{t}\left\|P u\left(t^{\prime}, \cdot\right)\right\|_{0} d t^{\prime} \\
& \quad \text { for any } \quad t \in[0, T], \quad u \in C_{0}^{\infty}\left([0, T] \times \mathbb{R}^{n}\right),
\end{aligned}
$$

then $P$ is regularly hyperbolic in $[0, T] \times \mathbb{R}^{n}$.

First, we will extend this theorem as follows.

Theorem 2.2. Let $d$ be an integer, $1 \leqq d \leqq m$, and assume that the following inequality holds:

$$
\begin{gathered}
\int_{0}^{t}\left\|\left\langle\hat{\xi}, D_{x}\right\rangle^{m-d} u\left(t^{\prime}, \cdot\right)\right\|_{0} d t^{\prime} \leqq C_{0} \int_{0}^{t}\left(t-t^{\prime}\right)^{d}\left\|P u\left(t^{\prime}, \cdot\right)\right\|_{0} d t^{\prime} \\
\text { for any } t \in[0, T], u \in C_{0}^{\infty}\left(V_{[0, T]}\right) .
\end{gathered}
$$

Here, $\left\langle\hat{\xi}, D_{x}\right\rangle=\sum_{j=1}^{n} \widehat{\xi}_{j} D_{x_{j}}, \widehat{\xi} \in S^{n-1}=\left\{\xi \in \mathbb{R}^{n} ;|\xi|=1\right\}$. Then, there exists 
a positive constant $\delta$ which depends only on $P_{m}, C_{0}$ and independent of $\hat{\xi}$ such that the following holds:

For any $(\hat{t}, \hat{x}) \in V_{[0, T]}$ and for any $\tau_{j}(j=1, \cdots, p)$ which are distinct characteristic roots at $(\hat{t}, \hat{x} ; \hat{\xi})$ with multiplicity $r_{j}$ and satisfy $\max _{j, k}\left|\tau_{j}-\tau_{k}\right| \leqq \delta$, there holds $\sum_{j=1}^{p} r_{j} \leqq d$.

Especially $P$ has no characteristic root whose multiplicity is larger than $d$.

Next we consider some weaker inequalities. That is, for non-negative integers $p, d$, and $\hat{\xi} \in S^{n-1}$,

$$
\begin{array}{cc}
(\mathrm{I}-p, d)_{\bar{\xi}} & \int_{0}^{t}\left\|\left\langle\hat{\xi}, D_{x}\right\rangle^{m-d} u\left(t^{\prime}, \cdot\right)\right\|_{0} d t^{\prime} \leqq C \int_{0}^{t}\left(t-t^{\prime}\right)^{p}\left\|P u\left(t^{\prime}, \cdot\right)\right\|_{0} d t^{\prime}, \\
(\mathrm{II}-p, d)_{\hat{\xi}} & \int_{0}^{t}\left\|\left\langle\hat{\xi}, D_{x}\right\rangle^{m-d} u\left(t^{\prime}, \cdot\right)\right\|_{0}^{2} d t^{\prime} \leqq C \int_{0}^{t}\left(t-t^{\prime}\right)^{p}\left\|P u\left(t^{\prime}, \cdot\right)\right\|_{0}^{2} d t^{\prime} \\
\text { for any } t \in[0, T], u \in C_{0}^{\infty}\left(V_{[0, T]}\right) .
\end{array}
$$

Further, we also consider the following inequality for integers $p, q$.

$$
\begin{aligned}
& (\mathrm{III}-p, q) \quad\|u\|_{H^{p}\left(V_{[0, t]}\right)} \leqq C\|P u\|_{H^{q}\left(V_{[0, t]}\right)} \\
& \text { for any } t \in[0, T], u \in C_{0}^{\infty}\left(V_{[0, T]}\right) .
\end{aligned}
$$

Remark 2.3. The difference between (I) and (II) is that of $L^{1}$ norm and $L^{2}$-norm w.r.t. $t$. The example which satisfies (II) but doesn't satisfy (I) is given later.

Theorem 2. 4. We assume that

(1) The C.P. for $P$ is well-posed and has a finite propagation speed in $V_{[0, T]}$.

(2) $P$ has a characteristic root $\hat{\tau}$ of multiplicity $r$ at $(\hat{t}, \hat{x} ; \hat{\xi})$ $\in V_{[0, T]} \times S^{n-1}$. If the inequality $(\mathrm{I}-p, d)_{\xi}(\operatorname{resp} \text {. (II }-p, d)_{\hat{\xi}}$, $\left.(\mathrm{III}-p, q)\right)$ holds, then $r \leqq 2 d-p \quad\left(\right.$ resp. $\left.r \leqq 2 d-\frac{p}{2}, r \leqq 2(m-p+q)\right)$ when $0<\hat{t}<T$, $r \leqq 3 d-2 p \quad($ resp. $r \leqq 3 d-p, r \leqq 3(m-p+q))$ when $\hat{t}=0$ or $T$. 
Remark 2.5. (a) Even if $\hat{t}=0$ or $T$, if $P_{m}$ can be extended as a hyperbolic operator with $C^{\infty}$-coefficients in a neighborhood of $(\hat{t}, \hat{x})$, then there hold the same results as in the case $0<\hat{t}<T$.

(b) In (III- $p, q)$, we may exchange $\|u\|_{H^{p}\left(V_{[0, \ell]}\right)}$ for $\int_{0}^{t}\left\|\left\langle\hat{\xi}, D_{x}\right\rangle^{p} u\left(t^{\prime}, \cdot\right)\right\|_{0} d t^{\prime}$ when $p \geqq 0$.

$(I-d, d)$ is the same as (2.1.2), and the result is $r \leqq d$, which coincides with the result in Theorem 2.2. On the other hand, in the case $(\mathrm{II}-d, d)$, the results are $r \leqq \frac{3}{2} d$ (when $0<\hat{t}<T$ ) and $r \leqq 2 d$ (when $\hat{t}=0$ or $T$ ). This difference actually occurs.

Example 2.6. We consider $P=D_{t}^{2}-t D_{x}^{2}+a(t, x) D_{t}+b(t, x) D_{x}$ $+c(t, x)\left(a, b, c \in \mathscr{B}^{\infty}([0, T] \times \boldsymbol{R})\right)$. Let $d$ be a positive integer. Then, we have the following energy inequality for $P^{d}$ :

For any non-negative integer $p$ and any real number s, there exists a positive constant $C_{p, s}$ such that

$$
\begin{aligned}
& \sum_{j=0}^{p+d}\left\|D_{t}^{j} u(t, \cdot)\right\|_{s+d-j}^{2} \\
& \leqq \\
& \quad C_{p, s}\left\{\sum_{j=0}^{p} \int_{0}^{t}\left(t-t^{\prime}\right)^{d-1}\left\|D_{t}^{j} P^{d} u\left(t^{\prime}, \cdot\right)\right\|_{s-j}^{2} d t^{\prime}\right. \\
& \quad+\sum_{j=0}^{p+d}\left\|D_{t}^{j} u(0, \cdot)\right\|_{s+d+1-j}^{2} \\
& \left.\quad+\sum_{h=1}^{d-1} t^{h} \sum_{j=0}^{p+d+h}\left\|D_{t}^{j} u(0, \cdot)\right\|_{s+d+h+1-j}^{2}\right\} \\
& \quad \text { for any } t \in[0, T], u \in C_{0}^{\infty}\left(\mathbb{R}^{2}\right) .
\end{aligned}
$$

Especially, we have

$$
\begin{gathered}
\sum_{j=0}^{m-d} \int_{0}^{t}\left\|D_{t}^{j} u\left(t^{\prime}, \cdot\right)\right\|_{m-d-j}^{2} d t^{\prime} \leqq C \int_{0}^{t}\left(t-t^{\prime}\right)^{d}\left\|P^{d} u\left(t^{\prime}, \cdot\right)\right\|_{0}^{2} d t^{\prime} \\
\text { for any } t \in[0, T], u \in C_{0}^{\infty}([0, T] \times \mathbb{R}),
\end{gathered}
$$

where $m=$ ord. $P^{d}=2 d$. Thus, for $P^{d}$, (II $\left.-d, d\right)_{\hat{\xi}}$ holds for any $\hat{\xi}$, but, by the result of Theorem 2.4., $(\mathrm{I}-d, d)_{\hat{\xi}}$ never holds. The proof of (2.1.3) is given in Appendix I. 


\section{$\S 2.2$. Proof of Theorem 2.2}

We use the method of Ivrii-Petkov [7; Theorem 1.1].

Let $\hat{\tau} \in \boldsymbol{R}, \mu>0, z=\hat{\tau}-i \mu$. And put

$$
u_{\rho}(t, x)=v(t, x) \exp \{i \rho(z t+\langle\hat{\xi}, x\rangle)\},
$$

where $v \in C_{0}^{\infty}\left(V_{[0, T]}\right), \rho>0$. We substitute $u_{\rho}$ for $u$ in $(2.1 .2)$, then

$$
\begin{gathered}
\int_{0}^{t}\left(t-t^{\prime}\right)^{d}\left\|P u_{\rho}\left(t^{\prime}, \cdot\right)\right\|_{0} d t^{\prime} \\
\leqq \int_{0}^{t}\left(t-t^{\prime}\right)^{d} \cdot \rho^{m} e^{\mu \rho t^{\prime}}\left(\int \mid P_{m}\left(t^{\prime}, x ; z, \hat{\xi}\right) v\left(t^{\prime}, x\right)\right. \\
\left.+\left.O\left(\rho^{-1}\right)\right|^{2} d x\right)^{1 / 2} d t^{\prime} \\
\leqq \rho^{m} \int_{0}^{t}\left(t-t^{\prime}\right)^{d} e^{\mu \rho t^{\prime}}\left\{\left(\int\left|P_{m}(t, x ; z, \hat{\xi}) v(t, x)\right|^{2} d x\right)^{1 / 2}\right. \\
\left.+O\left(t-t^{\prime}\right)+O\left(\rho^{-1}\right)\right\} d t^{\prime} .
\end{gathered}
$$

Here, we have

$$
\int_{0}^{t}\left(t-t^{\prime}\right)^{h} \cdot e^{\mu \rho t^{\prime}} d t^{\prime} \leqq h !(\mu \rho)^{-h-1} e^{\mu \rho t} \quad(h=0,1, \cdots) .
$$

So,

$$
\begin{aligned}
& \int_{0}^{t}\left(t-t^{\prime}\right)^{d}\left\|P u_{\rho}\left(t^{\prime}, \cdot\right)\right\|_{0} d t^{\prime} \\
& \quad \leqq \rho^{m-d-1} e^{\mu \rho t}\left\{d ! \mu^{-d-1}\left\|P_{m}(t, \cdot ; z, \hat{\xi}) v(t, \cdot)\right\|_{0}+O\left(\rho^{-1}\right)\right\}
\end{aligned}
$$

On the other hand, by $|\hat{\xi}|=1$, we have for fixed $t>0$,

$$
\begin{aligned}
& \int_{0}^{t}\left\|\left\langle\hat{\xi}, D_{x}\right\rangle^{m-d} u_{\rho}\left(t^{\prime}, \cdot\right)\right\|_{0} d t^{\prime} \\
& \geqq \rho^{m-d} \int_{0}^{t} e^{\mu \rho t^{\prime}}\left\{\left(\int|v(t, x)|^{2} d x\right)^{1 / 2}-O\left(t-t^{\prime}\right)-O\left(\rho^{-1}\right)\right\} d t^{\prime} \\
& \geqq \rho^{m-d-1} \mu^{-1}\left(e^{\mu \rho t}-1\right)\left\{\left(\int|v(t, x)|^{2} d x\right)^{1 / 2}-O\left(\rho^{-1}\right)\right\} .
\end{aligned}
$$

Thus, by letting $\rho \rightarrow+\infty$, we have

$$
C_{0} d ! \mu^{-d}\left\|P_{m}(t, \cdot ; z, \hat{\xi}) v(t, \cdot)\right\|_{0} \geqq\|v(t, \cdot)\|_{0}
$$




$$
\text { for any } t \in(0, T], \quad v \in C_{0}^{\infty}\left(V_{[0, T]}\right) \text {. }
$$

From this, we get

$$
\begin{aligned}
& \left|P_{m}(\hat{t}, \hat{x} ; \hat{\tau}-i \mu, \hat{\xi})\right| \geqq\left(C_{0} d !\right)^{-1} \mu^{d} \\
& \quad \text { for any } \mu>0, \quad(\hat{t}, \hat{x} ; \hat{\tau}) \in V_{[0, T]} \times \mathbb{R} .
\end{aligned}
$$

Now, let $(\hat{t}, \hat{x}) \in V_{[0, T]}$, and assume that $\tau_{j}(j=1, \cdots, p)$ are distinct characteristic roots at $(\hat{t}, \hat{x} ; \hat{\xi})$ with multiplicity $r_{j}$. Further we assume $\sum_{j=1}^{p} r_{j} \geqq d+1$ and we put $A=\max _{2 \leqq j \leqq p}\left|\tau_{1}-\tau_{j}\right|$. We have only to prove that there exists a positive constant $\delta$ which is independent of $(\hat{t}, \hat{x} ; \hat{\xi})$ and $\tau_{j}(1 \leqq j \leqq p)$ such that $A \geqq \delta$. We may assume $A \leqq 1$.

We can write $P_{m}(\hat{t}, \hat{x} ; \tau, \hat{\xi})=\prod_{j=1}^{p}\left(\tau-\tau_{j}\right)^{r_{j}} \cdot f(\tau)$, where $f(\tau)$ is a polynomial whose coefficients can be bounded by a constant independent of $(\hat{t}, \hat{x} ; \hat{\xi})$ and $\tau_{j}(1 \leqq j \leqq p)$, because the characteristic roots of $P_{m}$ are bounded. Now, we substitute $\tau_{1}$ for $\hat{\tau}$ in $(2.2 .2)$, then we get

$$
\mu^{r_{1}} \prod_{j=2}^{p}\left|\tau_{1}-\tau_{j}-i \mu\right|^{r_{j}}\left|f\left(\tau_{1}-i \mu\right)\right| \geqq\left(C_{0} d !\right)^{-1} \mu^{d}
$$

Thus, we have

$$
\mu^{d} \leqq C \mu^{r_{1}}(A+\mu)^{r_{2}+\cdots+r_{p}} \quad \text { for any } \quad \mu \in(0,1],
$$

where $C$ is a constant independent of $(\hat{t}, \widehat{x} ; \hat{\xi})$ and $\tau_{j}(1 \leqq j \leqq p)$. We take $\mu=A$, then

$$
A^{d} \leqq C \cdot 2^{m} \cdot A^{r_{1}+\cdots+r_{p}} .
$$

So, by $r_{1}+\cdots+r_{p}-d \geqq 1$, we have

$$
A \geqq\left(C \cdot 2^{m}\right)^{-1} \text { Q.E.D. }
$$

\section{$\S 2.3$. Proof of Theorem 2.4}

We need the following theorems.

Theorem 2.7. Let $\Omega$ be an open neighborhood of the origin in $\mathbb{R}^{n+1}$ and put $\Omega_{ \pm}=\{(t, x) \in \Omega ; \pm t \geqq 0\}$. We assume that $P_{m}$ has a characteristic root $\hat{\tau}$ at $(0,0 ; \hat{\xi}) \quad\left(\hat{\xi} \in \mathbb{R}^{n}-\{0\}\right)$.

(1) If $P_{m}$ is hyperbolic in $\Omega$, then 


$$
P_{m,(k, \beta)}^{(j, \alpha)}(0,0 ; \hat{\tau}, \hat{\xi})=0 \quad \text { for } \quad j+|\alpha|+k+|\beta|<r .
$$

(2) If $P_{m}$ is hyperbolic in $\Omega_{ \pm}$, then

$$
P_{m,(k, \beta)}^{(j, \alpha)}(0,0 ; \hat{\tau}, \hat{\xi})=0 \quad \text { for } \quad j+|\alpha|+2 k+|\beta|<r .
$$

Proof. We get the result from Lemma 1.3.1 in [5] by setting

$$
\begin{aligned}
& f\left(t, s_{1}, \cdots, s_{2 n+1}\right) \\
& = \begin{cases}P_{m}\left(s_{1}, \cdots, s_{n+1} ; t+\hat{\tau}, s_{n+2}+\hat{\xi}_{1}, \cdots, s_{2 n+1}+\hat{\xi}_{n}\right) & (\text { case }(1)), \\
P_{m}\left( \pm s_{1}^{2}, s_{2}, \cdots, s_{n+1} ; t+\hat{\tau}, s_{n+2}+\hat{\xi}_{1}, \cdots, s_{2 n+1}+\hat{\xi}_{n}\right) & (\text { case (2)). }\end{cases} \\
& \text { Q.E.D. }
\end{aligned}
$$

The next theorem plays the key part in our proof.

Theorem 2.8. ([7; Theorem 4.1]) We devide the variables as

$$
\begin{aligned}
& x=\left(x^{(1)}, x^{(2)}\right), \xi=\left(\xi^{(1)}, \xi^{(2)}\right), \\
& x^{(1)}=\left(x_{1}, \cdots, x_{\nu}\right), x^{(2)}=\left(x_{\nu+1}, \cdots, x_{n}\right)(0 \leqq \nu \leqq n-1) \text { etc. }
\end{aligned}
$$

Let $p \geqq q>0$ be rational numbers, $r$ be a positive integer and $(\hat{t}, \hat{x})$ $\in V_{[0, T]}$. Further we assume

$$
\begin{aligned}
& P_{m}^{(r, 0)}\left(\hat{t}, \hat{x} ; 0,0, \xi^{(2)}\right) \neq 0 \text { for any } \xi^{(2)} \in \boldsymbol{R}^{n-\nu}-\{0\}, \\
& P_{m,(k, \beta)}^{(j, \alpha)}\left(\hat{t}, \hat{x} ; 0,0, \xi^{(2)}\right)=0 \text { for any } \xi^{(2)} \in \boldsymbol{R}^{n-\nu}, \\
& \text { if } j+|\alpha|+p\left(k+\left|\beta^{(1)}\right|\right)+q\left|\beta^{(2)}\right|<r .
\end{aligned}
$$

If the C.P. for $P$ is well-posed and has a finite propagation speed in $V_{[0, T]}$, and if $j+|\alpha|+p\left(k+\left|\beta^{(1)}\right|\right)+q\left|\beta^{(2)}\right|<r-h(1+p) \quad(h=1, \cdots, m)$, then

$$
P_{m-h,(k, \beta)}^{(j, \alpha)}\left(\hat{t}, \hat{x} ; 0,0, \xi^{(2)}\right)=0 \text { for any } \xi^{(2)} \in \boldsymbol{R}^{n-\nu} .
$$

Now, if $P_{m}$ has a characteristic root $\hat{\tau}$ of multiplicity $r$ at $(\hat{t}, \widehat{x} ; \hat{\xi})$ $\left(\hat{\xi} \in S^{n-1}\right)$, then by a suitable orthogonal transformation

$$
\begin{gathered}
\left(\begin{array}{l}
s=t \\
y=a(t-\hat{t})+A(x-\hat{x})+\hat{x}
\end{array}\right. \\
\left(a \in \boldsymbol{R}^{n}, A \text { is a orthogonal }(n \times n) \text {-matrix }\right),
\end{gathered}
$$

$\hat{\xi}, \hat{\tau}$ and $\left\langle\hat{\xi}, D_{x}\right\rangle$ are transformed into $e_{n}=(0, \cdots, 0,1), 0$ and $\left\langle e_{n}, D_{y}\right\rangle$ 
$=D_{y_{n}}$, respectively. So, we may assume that $\hat{\xi}=e_{n}, \hat{\tau}=0$.

From Theorems $0.3,2.7$ and 2.8 , we have

Corollary 2.9. If the C.P. for $P$ is well-posed and has a finite propagation speed in $V_{[0, T]}$ and $P_{m}$ has a characteristic root $\hat{\tau}=0$ with multiplicity $r$ at $\left(\hat{t}, \widehat{x} ; e_{n}\right)\left((\hat{t}, \widehat{x}) \in V_{[0, T]}\right)$, then for $h=0,1, \cdots, m$,

(1) when $0<\hat{t}<T$,

(2.3.1) $\quad P_{m, h,(k . \beta)}^{(j, a)}\left(\hat{t}, \widehat{x} ; 0, c_{n}\right)=0$ for $j+|\alpha|+k+|\beta|<r-2 h$,

(2) when $\hat{t}=0$ or $T$,

(2.3.2) $\quad P_{m-h,(k, \beta)}^{(j, \alpha)}\left(\hat{t}, \widehat{x} ; 0, e_{n}\right)=0$

$$
\text { for } j+|\alpha|+2\left(k+\beta_{1}+\cdots+\beta_{n-1}\right)+\beta_{n}<r-3 h .
$$

Now, we start the proof of Theorem 2.4. As is seen above, we may assume $(\hat{\tau}, \hat{\xi})=\left(0, e_{n}\right), \widehat{x}=0$.

(1) When $0<\hat{t}<T$, we consider the coordinate transformation

$$
\begin{cases}s=\rho(t-\hat{t}) & \\ y_{j}=\rho x_{j} & (j=1, \cdots, n-1) \\ y_{n}=\rho^{2} x_{n} & (\rho \geqq 1) .\end{cases}
$$

Under this transformation, $P$ is transformed into

$$
\begin{gathered}
P_{\rho}\left(s, y ; D_{s}, D_{y}\right)=P\left(s \rho^{-1}+\hat{t}, y_{1} \rho^{-1}, \cdots, y_{n-1} \rho^{-1}, y_{n} \rho^{-2}\right. \\
\left.\rho D_{s}, \rho D_{y_{1}}, \cdots, \rho D_{y_{n-1}}, \rho^{2} D_{y_{n}}\right) .
\end{gathered}
$$

Here, for sufficiently large $N$, we have

$$
\begin{gathered}
P_{\rho, m-h}(s, y ; \sigma, \eta)=P_{m-h}\left(s \rho^{-1}+\hat{t}, y_{1} \rho^{-1}, \cdots, y_{n-1} \rho^{-1}, y_{n} \rho^{-2}\right. \\
\left.\rho \sigma, \rho \eta_{1}, \cdots, \rho \eta_{n-1}, \rho^{2} \eta_{n}\right) \\
=\rho^{2(m-h)} \eta_{n}^{m-h} P_{m-n}\left(s \rho^{-1}+\hat{t}, y_{1} \rho^{-1}, \cdots, y_{n} \rho^{-2} ;\right. \\
\left.\frac{\sigma}{\rho \eta_{n}}, \frac{\eta_{1}}{\rho \eta_{n}}, \cdots, \frac{\eta_{n-1}}{\rho \eta_{n}}, 1\right) \\
=\rho^{2(m-h)} \eta_{n}^{m-n} \sum_{a_{n}=0, k+|\beta|<N} j \overline{j ! \alpha ! \hat{k} ! \overline{\beta !}}\left(s \rho^{-1}\right)^{k}\left(y_{1} \rho^{-1}\right)^{\beta_{1}} \ldots
\end{gathered}
$$




$$
\begin{aligned}
& \cdots\left(y_{n} \rho^{-2}\right)^{\beta_{n}}\left(\frac{\sigma}{\rho \eta}\right)^{j} \cdots\left(\frac{\eta_{n-1}}{\rho \eta_{n}}\right)^{\alpha_{n-1}} \\
& \times P_{m-h,(k, \beta)}^{(j, \alpha)}\left(\hat{t}, 0 ; 0, e_{n}\right)+Q_{m-h, \rho}(s, y ; \sigma, \eta) \\
= & \rho^{2 m-r} \sum_{\alpha_{n}=0, k+|\beta|<N} \frac{1}{j ! \alpha ! k ! \beta !} P_{m-h,(k, \beta)}^{(j, \alpha)}\left(\hat{t}, 0 ; 0, e_{n}\right) \\
& \times \rho^{r-2 h-k-|\beta|-\beta_{n}-j-|\alpha|} s^{k} y^{\beta} \sigma^{j} \eta^{\alpha} \eta_{n}^{m-h-j-|\alpha|}+Q_{m-h, \rho}(s, y ; \sigma, \eta),
\end{aligned}
$$

where $Q_{m-h, \rho}$ is a homogeneous polynomial of degree $m-h$ w.r.t. $(\sigma, \eta)$ with coefficients which are bounded in $C^{\infty}$ when $\rho \rightarrow+\infty$. By (2.3.1), we have $P_{\rho}=\rho^{2 m-r} R_{\rho}\left(s, y ; D_{s}, D_{y}\right)$, where $R_{\rho}(s, y ; \sigma, \eta)$ is a polynomial of degree $m$ w.r.t. $(\sigma, \eta)$ with coefficients which are bounded in $C^{\infty}$ when $\rho \rightarrow+\infty$.

Now, for $v \in C_{0}^{\infty}(B)$ ( $B$ is the open unit ball in $\boldsymbol{R}^{n+1}$ ), we put

$$
u_{\rho}(t, x)=v\left(\rho(t-\hat{t}), \rho x_{1}, \cdots, \rho x_{n-1}, \rho^{2} x_{n}\right) .
$$

Then, $u_{\rho} \in C_{0}^{\infty}\left(V_{[0, T]}\right)$ for sufficiently large $\rho$ and

$$
\left(P u_{\rho}\right)(t, x)=\left(P_{\rho} v\right)\left(\rho(t-\hat{t}), \rho x_{1}, \cdots, \rho^{2} x_{n}\right) .
$$

We substitute $u_{\rho}$ for $u$ in $(\mathrm{I}-p, d)_{\hat{\xi}},(\mathrm{II}-p, d)_{\hat{\xi}},(\mathrm{III}-p, q)$. Then, we have

$$
\begin{gathered}
\int_{0}^{t}\left\|D_{x_{n}}^{m-d} u_{\rho}\left(t^{\prime}, \cdot\right)\right\|_{0} d t^{\prime}=\rho^{2(m-d)} \int_{0}^{t}\left(\int \mid\left(D_{x_{n}}^{m-d} v\right)\left(\rho\left(t^{\prime}-\hat{t}\right)\right.\right. \\
\left.\left.\rho x_{1}, \cdots, \rho x_{n-1}, \rho^{2} x_{n}\right)\left.\right|^{2} d x\right)^{1 / 2} d t^{\prime} \\
=\rho^{2(m-d)-(n+3) / 2} \int_{-\hat{t} \hat{t}}^{\rho(t-\hat{t})}\left\|\left(D_{x_{n}}^{m-d} v\right)\left(t^{\prime}, \cdot\right)\right\|_{0} d t^{\prime}
\end{gathered}
$$

and

$$
\begin{aligned}
& \int_{0}^{t}\left(t-t^{\prime}\right)^{p}\left(\int\left|\left(P_{\rho} v\right)\left(\rho\left(t^{\prime}-\hat{t}\right), \rho x_{1}, \cdots, \rho^{2} x_{n}\right)\right|^{2} d x\right)^{1 / 2} d t^{\prime} \\
& =\rho^{2 m-r-(n+3) / 2} \int_{-\rho \hat{t}}^{\rho(t-\hat{t})}\left(t-\frac{t^{\prime}}{\rho}-\hat{t}\right)^{p}\left\|\left(R_{\rho} v\right)\left(t^{\prime}, \cdot\right)\right\|_{0} d t^{\prime} \\
& =\rho^{2 m-r-(n+3) / 2-p} \int_{-\rho \hat{t}}^{\rho(t-\hat{t})}\left(\rho(t-\hat{t})-t^{\prime}\right)^{p}\left\|\left(R_{\rho} v\right)\left(t^{\prime}, \cdot\right)\right\|_{0} d t^{\prime} .
\end{aligned}
$$

Thus, from $(\mathrm{I}-p, d)_{\hat{\xi}}$, by taking $t=\hat{t}+\rho^{-1}$ and letting $\rho \rightarrow+\infty$, we have $2(m-d)-\frac{n+3}{2} \leqq 2 m-r-p-\frac{n+3}{2}$, that is, $r \leqq 2 d-p$. 
We also have

$$
\begin{aligned}
& \int_{0}^{t}\left\|D_{x_{n}}^{m-d} u\left(t^{\prime}, \cdot\right)\right\|_{0}^{2} d t^{\prime} \\
& =\rho^{4(m-d)} \int_{0}^{t} \int\left|\left(D_{x_{n}}^{m-d} v\right)\left(\rho\left(t^{\prime}-\hat{t}\right), \rho x_{1}, \cdots, \rho^{2} x_{n}\right)\right|^{2} d x d t^{\prime} \\
& =\rho^{4(m-d)-(n+2)} \int_{-\rho \hat{t}}^{\rho(t-\hat{t})}\left\|\left(D_{x_{n}}^{m-d} v\right)\left(t^{\prime}, \cdot\right)\right\|_{0}^{2} d t^{\prime}
\end{aligned}
$$

and

$$
\begin{aligned}
& \int_{0}^{t}\left(t-t^{\prime}\right)^{p} \int\left|\left(P_{\rho} v\right)\left(\rho\left(t^{\prime}-\hat{t}\right), \rho x_{1}, \cdots, \rho^{2} x_{n}\right)\right|^{2} d x d t^{\prime} \\
& =\rho^{2(2 m-r)-(n+2)} \int_{-\rho \hat{t}}^{\rho(t-\hat{t})}\left(t-\frac{t^{\prime}}{\rho}-\hat{t}\right)^{p}\left\|\left(R_{\rho} v\right)\left(t^{\prime}, \cdot\right)\right\|_{0}^{2} d t^{\prime} \\
& =\rho^{2(2 m-r)-(n+2)-p} \int_{-\rho \hat{t}}^{\rho(t-\hat{t})}\left(\rho(t-\hat{t})-t^{\prime}\right)^{p} \\
& \quad \times\left\|\left(R_{\rho} v\right)\left(t^{\prime}, \cdot\right)\right\|_{0}^{2} d t^{\prime} .
\end{aligned}
$$

Thus, from (II- $p, d)_{\hat{\xi}}$, by taking $t=\hat{t}+\rho^{-1}$ and letting $\rho \rightarrow+\infty$, we have $4(m-d)-(n+2) \leqq 2(2 m-r)-(n+2)-p$, that is, $r \leqq 2 d-\frac{p}{2}$.

For the case (III- $p, q$ ), we use the following lemma.

Lemma 2.10. For $u \in C_{0}^{\infty}(B)$, we put

$$
\widetilde{u}(t, x)=u\left(\rho^{\sigma_{0}}(t-\hat{t}), \rho^{\sigma_{1}} x_{1}, \cdots, \rho^{\sigma_{n}} x_{n}\right),
$$

where $0 \leqq \sigma_{j} \leqq \sigma_{n}(j=1, \cdots, n-1)$. Then, for $t_{0} \in[0, T]$ and for an integer $h$,

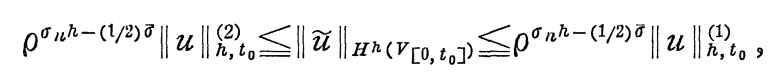

where

$$
\begin{aligned}
& \|u\|_{h, t}^{(1)}= \begin{cases}\|u\|_{H^{h}\left(B_{\rho}, t\right)} & (\text { when } h \geqq 0), \\
\sup _{w \in C_{0}^{\infty}\left(B_{\rho, t}\right)} \frac{\left|(u, w)_{L^{2}\left(B_{\theta}, t\right)}\right|}{\left\|D_{x_{n}}^{-h} w\right\|_{L^{2}\left(B_{\rho, t}\right)}} & (\text { when } h \leqq 0),\end{cases}
\end{aligned}
$$

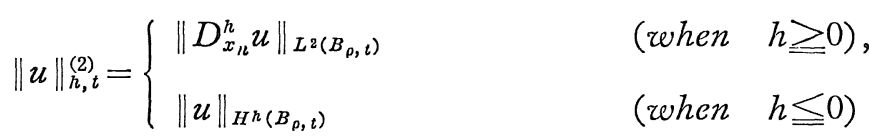

and

$$
B_{\rho, t}=B_{\left[-\rho \sigma_{0} \hat{t}, \rho \sigma_{0}(t-\hat{t})\right]}, \quad \bar{\sigma}=\sum_{j=0}^{n} \sigma_{j} .
$$


When $h$ is a positive integer, we can exchange

$$
\|\widetilde{u}\|_{H^{h}\left(V_{\left[0, t_{0}\right]}\right)} \quad \text { for } \quad \int_{0}^{t_{0}}\left\|D_{x_{n}}^{h} \widetilde{u}\right\|_{0} d t
$$

Proof of Lemma 2.10. When $h \geqq 0$,

$$
\begin{aligned}
& \|\tilde{u}\|_{H^{h}\left(V_{\left.\left[0, t_{0}\right]\right)}\right.}{ }^{2} \\
& \leqq \rho^{2 \sigma_{n} h} \sum_{j+|\alpha| \leqq n} \int_{0}^{t_{0}} \int \mid\left(D_{t}^{j} D_{x}^{\alpha} u\right)\left(\rho^{\sigma_{0}}(t-\hat{t}), \rho^{\sigma_{1}} x_{1}, \cdots\right. \\
& \left.\quad \cdots, \rho^{\sigma_{n}} x_{n}\right)\left.\right|^{2} d x d t \\
& =\rho^{2 \sigma_{1 l^{h}-\bar{\sigma}}} \sum_{j+|\alpha| \leqq n} \int_{-\rho^{\sigma_{0} \hat{t}}}^{\rho^{\sigma_{0}}\left(t_{0}-\hat{t}\right)} \int\left|\left(D_{t}^{j} D_{x} u\right)(t, x)\right|^{2} d x d t \\
& =\rho^{2 \sigma_{n} h-\bar{\sigma}}\left(\|u\|_{h, t_{0}}^{(1)}\right)^{2},
\end{aligned}
$$

and

$$
\begin{aligned}
& \|\widetilde{u}\|_{H^{n}\left(V_{\left[0, t_{0}\right]}\right)}{ }^{2} \geq \int_{0}^{t_{0}}\left\|D_{x_{n}}^{h} \widetilde{u}(t, \cdot)\right\|_{0}^{2} d t \\
& =\rho^{2 \sigma_{n^{h}}} \int_{0}^{t_{0}} \int\left|\left(D_{x_{n}}^{h} u\right)\left(\rho^{\sigma_{0}}(t-\hat{t}), \cdots, \rho^{\sigma_{n}} x_{n}\right)\right|^{2} d x d t \\
& =\rho^{2 \sigma_{n} h-\bar{\sigma}} \int_{-\rho^{\sigma_{0} \hat{t}}}^{\rho^{\sigma_{0}\left(t_{0}-\hat{t}\right)}} \int\left|\left(D_{x_{n}}^{h} u\right)(t, x)\right|^{2} d x d t \\
& =\rho^{2 \sigma_{n} h-\bar{\sigma}}\left(\|u\|_{h, t_{0}}^{(2)}\right)^{2} .
\end{aligned}
$$

When $h \leqq 0$,

$$
\begin{aligned}
& \|\widetilde{u}\|_{H^{h}\left(V_{\left[0, t_{0}\right]}\right)} \\
& =\sup _{\widetilde{w} \in C_{0}^{\infty}\left(V_{\left[0, t_{0}\right]}\right)} \frac{\left|(\widetilde{u}, \widetilde{\widetilde{w}})_{L^{2}\left(V_{\left[0, t_{0}\right]}\right)}\right|}{\|\widetilde{\widetilde{w}}\|_{H^{-h}\left(V_{\left[0, t_{0}\right]}\right)}} .
\end{aligned}
$$

Here, we take $\widetilde{w}(t, x)=w\left(\rho^{\sigma_{0}}(t-\hat{t}), \cdots, \rho^{\sigma_{n}} x_{n}\right)$ for $w \in C_{0}^{\infty}\left(B_{\rho, t_{0}}\right)$. Because $\widetilde{u}$ is the same form as $\widetilde{w}$, we have, by using the result when $h \geqq 0$ for $\widetilde{w}$,

$$
\begin{aligned}
& \|\widetilde{u}\|_{H^{h}\left(V_{\left[0, t_{0}\right]}\right)}=\sup _{w \in C_{0}^{\infty}\left(B_{\left.\rho, t_{0}\right)}\right.} \frac{\left|\int_{0}^{t_{0}} \int \widetilde{u}(t, x) \overline{\widetilde{w}(t, x)} d x d t\right|}{\|\widetilde{\widetilde{w}}\|_{H^{-h}\left(V_{\left[0, t_{0}\right]}\right)}} \\
& \leqq \rho^{-\bar{\sigma}+\sigma_{n} h+(1 / 2) \bar{\sigma}} \sup _{w \in C_{0}^{\infty}\left(B_{\rho, t}\right)} \frac{\left|(u, w)_{L^{2}\left(B_{\rho, t_{0}}\right)}\right|}{\left\|D_{x_{n}}^{-h} w\right\|_{L^{2}\left(B_{\rho}, t_{0}\right)}}
\end{aligned}
$$




$$
=\rho^{\sigma} \imath^{h-(1 / 2) \bar{\sigma}}\|u\|_{h, t_{0}}^{(1)} .
$$

and

$$
\begin{aligned}
& \|u\|_{H^{h}\left(V_{\left[0, t_{0}\right]}\right)} \\
& \geq \rho^{-\bar{\sigma}+\sigma_{n} h+(1 / 2) \bar{\sigma}} \sup _{w \in C_{0}^{\infty}\left(B_{\left.\rho, t_{0}\right)}\right.} \frac{\left|(u, w)_{L^{2}\left(B_{\rho}, t_{0}\right)}\right|}{\|w\| \|_{H^{-h}\left(B_{\rho, t_{0}}\right)}} \\
& \quad=\rho^{\sigma_{\eta} h-(1 / 2) \bar{\sigma}}\|u\|_{h, t_{0}}^{(2)} .
\end{aligned}
$$

We return to the proof in the case (III- $-p, q)$. We have

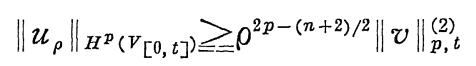

and

$$
\left\|P u_{\rho}\right\|_{H^{q}\left(V_{[0, t]}\right)} \leqq \rho^{2 m-r+2 q-(n+2) / 2}\left\|R_{\rho} v\right\|_{q, t}^{(1)}
$$

Thus, by taking $t=\hat{t}+\rho^{-1}$ and letting $\rho \rightarrow+\infty$, from (III- $\rho, q$ ), we have $2 p-\frac{n+2}{2} \leqq 2 m-r+2 q-\frac{n+2}{2}$, that is, $r \leqq 2(m+q-p)$.

(2) When $\hat{t}=0$ or $T$, we perform the coordinate transformation

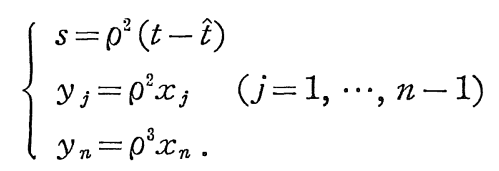

As in the case (1), under this transformation, $P$ is transformed into $P_{\rho}=\rho^{3 m-r} R_{\rho}$, where $R_{\rho}$ is the same as in the case (1).

When $\hat{t}=0$, we take

$$
\begin{gathered}
u_{\rho}(t, x)=v\left(\rho^{2} t, \rho^{2} x_{1}, \cdots, \rho^{2} x_{n-1}, \rho^{3} x_{n}\right) \\
\text { for } v \in C_{0}^{\infty}\left(B_{[0,1]}\right),
\end{gathered}
$$

and, when $\hat{t}=T$, we take

$$
\begin{gathered}
u_{\rho}(t, x)=v\left(\rho^{2}(t-T), \rho^{2} x_{1}, \cdots, \rho^{3} x_{n}\right) \\
\text { for } \quad v \in C_{0}^{\infty}\left(B_{[-1,0]}\right) .
\end{gathered}
$$

Just as in the case (1), we get the desired results.

Q.E.D. 


\section{Chapter 3. The Effect of Lower Order Terms}

\section{$\S 3.1$. Results and Examples}

In this chapter, we will consider the effect of lower order terms to energy inequalities. Many authors have investigated weakly hyperbolic equations. And as a method of proving the well-posedness, some showed energy inequalities, in which the order of differentiation depends on lower order terms. ([17], [12], [16], [14], [15], [9], [21], [6], [22], etc.) Others constructed parametrices or fundamental solutions, which belong to symbol classes depending on lower order terms. ([1], [2], [24], [25], [11], [13], etc.) As is said in Introduction, these suggest that the regularity of solutions may get worse depending on lower order terms. The following example illustrates clearly that this phenomenon actually occurs for some operators.

Example 3.1 (cf. [4], [17]). We consider the operator

$$
P=D_{t}^{2}+t^{k} D_{t} D_{x}+a i t^{k-1} D_{x},
$$

where $a=N(k+1)+2$ and $k, N$ are positive integers. The C.P. for $P$ is well-posed for any $a$, and we can explicitly find $u$ which satisfies

$$
\left\{\begin{array}{l}
P u=f(x) \\
\left.D_{t}^{j} u\right|_{t=0}=0(j=0,1), \text { where } f \in \mathscr{D}^{\prime}(\boldsymbol{R}),
\end{array}\right.
$$

in the form

$$
u(t, x)=\sum_{j=0}^{N} A_{j} t^{j(k+1)+2}\left(\partial_{x}^{j} f\right)(x),
$$

where $A_{j}(0 \leqq j \leqq N)$ are positive constants independent of $f$. So, in this unique solution, $\left(\partial_{x}^{j} f\right)(x)$ actually appears and when $a \rightarrow+\infty, N \rightarrow$ $+\infty$. (This example is a variant of the example stated in [17], and can be proved by putting (3.1.3) into (3.1.2).)

In this chapter, we consider the following energy inequality, and we study the relation between $(q-p)$ and $P_{m-1}$. (If the C. P. for $P$ is well-posed, this inequality holds for some $p, q$. See [7; Lemma 2.1].) 


$$
\begin{aligned}
& \|u\|_{H^{p}\left(U_{t}\right)} \leqq C\|P u\|_{H^{q}\left(U_{t}\right)} \\
& \quad \text { for } t \in\left[-T_{0}, T_{1}\right], u \in C_{0}^{\infty}\left(U_{T_{1}}\right) .
\end{aligned}
$$

Here, $\left[-T_{0}, T_{1}\right]=[0, T]$ (we call case (i)) or $[-T, 0]$ (case (ii)) $(T>0), p, q$ are integers, $U$ is an open neighborhood of the origin in $\boldsymbol{R}^{n+1}, U_{t}=U_{\left[-T_{0}, t\right]}\left(t \in\left[-T_{0}, T_{1}\right]\right)$.

About this problem, Ivrii-Petkov [7; Theorem 3] proved the following result.

Theorem 3.2. If we assume

(i) $P_{m}(0,0 ; \hat{\tau}, \hat{\xi})=0 \quad\left((\hat{\tau}, \hat{\xi}) \in \mathbb{R} \times\left(\mathbb{R}^{n}-\{0\}\right)\right)$,

(ii) $\operatorname{grad}_{(t, x ; \tau, \xi)} P_{m}(0,0 ; \hat{\tau}, \hat{\xi})=0$,

(iii) the fundamental matrix of $P_{m}$ at $(0,0 ; \hat{\tau}, \hat{\xi})$,

$$
F_{P_{m}}(0,0 ; \hat{\tau}, \hat{\xi})=\left(\begin{array}{cc}
\left(\partial_{\xi_{i}} \partial_{x_{j}} P_{m}\right)_{0 \leqq i, j \leqq n} & \left(\partial_{\xi_{i}} \partial_{\xi_{j}} P_{m}\right)_{0 \leqq i, j \leqq n} \\
-\left(\partial_{x_{i}} \partial_{x_{j}} P_{m}\right)_{0 \leqq i, j \leqq n} & -\left(\partial_{x_{i}} \partial_{\xi_{j}} P_{m}\right)_{0 \leqq i, j \leqq n}
\end{array}\right),
$$

where $x_{0}=t, \xi_{0}=\tau$, has non-zero real eigenvalues $\pm \mu$, (Hörmander [5; Corollary 1.4.7] called such operators effectively hyperbolic),

(iv) the inequality (3.1.4) holds, then

$$
\frac{\left|\operatorname{Im} P_{m-1}^{s}(0,0 ; \hat{\tau}, \hat{\xi})\right|}{|\mu|} \leqq C \cdot n \cdot(q+m-p) .
$$

Here, $C$ is an absolute constant, and $P_{m-1}^{s}$ is the subprincipal symbol of $P$.

We will show some results of the same type for some operators.

First, we consider the operators of the following form in $U_{T_{1}}$.

$$
\begin{aligned}
P(t, x ; \tau, \xi)= & Q_{m}\left(t, x ; \tau, \xi^{\prime}, t^{k} \xi_{n}\right) \\
& +\sum_{n=1}^{m} t^{-h} Q_{m-h}\left(t, x ; \tau, \xi^{\prime}, t^{k} \xi_{n}\right),
\end{aligned}
$$

where $Q_{j}$ is a homogeneous polynomial of degree $j$ w.r.t. $(\tau, \xi)$ with $C^{\infty}$-coefficients and $m, k$ are positive integers and $m \geqq 2$.

We assume 


$$
\begin{aligned}
& \text { (i) the coefficients of } Q_{m} \text { are real-valued, } \\
& \text { (ii) } Q_{m}(0,0 ; \hat{\tau}, 0,1)=0 \quad(\hat{\tau} \in \boldsymbol{R}) \\
& \text { (iii) }\left(\partial_{-} Q_{m}\right)(0,0 ; \hat{\tau}, 0,1) \neq 0 .
\end{aligned}
$$

Theorem 3.3. Under the above situation, there exists a constant $C_{m, k}$ which depends only on $m$ and $k$, such that if the inequality (3.1.4) holds, then

(3.1.8) $\pm \operatorname{Im}$

$$
\begin{gathered}
\frac{Q_{m-1}(0,0 ; \hat{\tau}, 0,1)+\frac{k}{2 i} \cdot \hat{\tau} \cdot\left(\partial_{\tau}^{2} Q_{m}\right)(0,0 ; \hat{\tau}, 0,1)}{\left(\partial_{\tau} Q_{m}\right)(0,0 ; \hat{\tau}, 0,1)} \\
\leqq C_{m, k}(q+m-1-p),
\end{gathered}
$$

where we take + in the case (i) and - in the case (ii).

A preciser result for $C_{m, k}$ is given in (3.2.14), but this value is far from the best possible. (cf. [1], [2], [8], [24], [25], [13].)

Remark 3.4. (a) If we assume that $P_{m}(t, x ; \tau, \xi)=Q_{m}\left(t, x ; \tau, \xi^{\prime}\right.$, $\left.t^{k} \xi_{n}\right)$, where $Q_{m}(t, x ; 1,0,0) \neq 0$, and that the coefficients of $P$ are $C^{\infty}$. functions, and that the C. P. for $P$ is well-posed with a finite propagation speed, then $P$ must be written in the form (3.1.6). (This follows from Theorem 4.1 in [7]. (See Theorem 2.8 in $\S 2.3$.$) )$

(b) By a result in Chapter 2 , if $Q_{m}(t, x ; 1,0,0) \neq 0$, and that the coefficients of $P$ are $C^{\infty}$, then $(q+m-1-p) \geqq 0$. (In Chapter 2, we have assumed that the C.P. for $P$ is well-posed and has a finite propagation speed. But we use these conditions only in Theorem 2.8. If $P$ has the form (3.1.6), the conclusion of Theorem 2.8 is satisfied without these conditions. So, we have $q+m-1-p \geqq 0$.)

(c) The condition that $P$ can be written in the form (3.1.6) is invariant under a coordinate transformation of the form

$$
\left\{\begin{array}{l}
s=t \\
y_{j}=f_{j}(x, t) \quad(j=1, \cdots, n-1) \\
y_{n}=f_{n}\left(x_{n}\right)+\frac{1}{k+1} t^{k+1} h(t, x)
\end{array}\right.
$$

where $f_{j}(0,0)=0(j=1, \cdots, n-1), f_{n}(0)=0$. Under this transformation, 
if we write the symbol of transformed operator as

$$
\widetilde{P}=\widetilde{Q}_{m}\left(s, y ; \sigma, \eta^{\prime}, s^{k} \eta_{n}\right)+\sum_{h=1}^{m} s^{-h} \widetilde{Q}_{m-h}\left(s, y ; \sigma, \eta^{\prime}, s^{k} \eta_{n}\right)
$$

then

$$
\left\{\begin{array}{l}
\widetilde{Q}_{m}(0,0 ; \hat{\sigma}, 0,1)=0, \\
\left(\partial_{\sigma} \widetilde{Q}_{m}\right)(0,0 ; \hat{\sigma}, 0,1) \neq 0, \\
\frac{\widetilde{Q}_{m-1}(0,0 ; \hat{\sigma}, 0,1)+\frac{k}{2 i} \cdot \hat{\sigma} \cdot\left(\partial_{\sigma}^{2} \widetilde{Q}_{m}\right)(0,0 ; \hat{\sigma}, 0,1)}{\left(\partial_{\sigma} \widetilde{Q}_{m}\right)(0,0 ; \hat{\sigma}, 0,1)} \\
=\frac{Q_{m-1}(0,0 ; \hat{\tau}, 0,1)+\frac{k}{2 i} \cdot \hat{\tau} \cdot\left(\partial_{\tau}^{2} Q_{m}\right)(0,0 ; \hat{\tau}, 0,1)}{\left(\partial_{\tau} Q_{m}\right)(0,0 ; \hat{\tau}, 0,1)},
\end{array}\right.
$$

where $\hat{\sigma}=f_{n}^{\prime}(0) \hat{\tau}-h(0,0)$. So, the quantity in the left-hand side of (3.1.8) has some invariance for $P$. (These are obtained by straight calculations, so the proof is left to readers.) This quantity appeared also in the arguments of Nakamura-Uryu [13] (their $m_{i}$ 's), and the class to which their parametrix belongs is determined by this quantity.

We give some examples of operators for which the C.P. is wellposed and can be written in the form (3.1.6).

Example 3.5. (1) Let $Q(t, x ; \tau, \xi)$ be strictly hyperbolic, and put

$$
P(t, x ; \tau, \xi)=\sum_{n=0}^{m} t^{-h} Q_{m-h}\left(t, x ; \tau, t^{k_{1}} \xi_{1}, \cdots, t^{k_{n}} \xi_{n}\right)
$$

where $k_{j}$ are non-negative integers. If the coefficients of $P$ are $C^{\infty}$, then the C.P. for $P$ is well-posed. ([20], etc.)

(2) As a special case of (1), we consider

$$
P=D_{t}^{2}-2 a(t, x) t^{k} D_{t} D_{x}+b(t, x) t^{2 k} D_{x}^{2}+c(t, x) t^{k-1} D_{x},
$$

where $a, b, c \in C^{\infty}\left(\left[-T_{0}, T_{1}\right] \times \mathbb{R}\right)$, and $a, b$ are real-valued, and $a(t, x)^{2}$ $-b(t, x)>0$ on $\left[-T_{0}, T_{1}\right] \times \mathbb{R}$. In this case, we can take $\hat{\tau}=a(0,0)$ $\pm \sqrt{a(0,0)^{2}-b(0,0)}$, and then

$$
\left\{\begin{array}{l}
Q_{m-1}(0,0 ; \hat{\tau}, 0,1)=c(0,0) \\
\left(\partial_{\tau} Q_{m}\right)(0,0 ; \hat{\tau}, 0,1)= \pm 2 \sqrt{a(0,0)^{2}-b(0,0)} \\
\left(\partial_{\tau}^{2} Q_{m}\right)(0,0 ; \hat{\tau}, 0,1)=2
\end{array}\right.
$$


So, if (3.1.4) holds, we have

$$
\frac{|\operatorname{Im} c(0,0)-k a(0,0)|}{\sqrt{a(0,0)^{2}-b(0,0)}} \leqq C_{k}(q+1-p)+k,
$$

where $C_{k}$ is a constant which depends only on $k$. The left-hand side of this inequality coincides with that of (3.1.5), when $k=1$.

The operators we considered above have characteristic roots which coincide with each other when $t=0$ with a finite order. Next, we consider the case with infinite degeneracy.

Let

$$
\alpha(t)=\mid t_{1}^{\mu} \exp \left(-B(t)|t|^{-\omega}\right) \quad \text { on } \quad\left[-T_{0}, T_{1}\right]
$$

where $\mu \in \boldsymbol{R}, \omega$ is a positive integer and $B \in C^{\infty}\left[-T_{0}, T_{1}\right], B(0)>0$. Note that $\alpha \in C^{\infty}\left[-T_{0}, T_{1}\right]$ and $\alpha$ is flat at $t=0$. Further,

$$
\alpha^{\prime}(t) / \alpha(t)=b(t)|t|^{-\omega-1},
$$

where $b \in C^{\infty}\left[-T_{0}, T_{1}\right], b(0)= \pm \omega B(0)$ ( + in the case (i), - in the case (ii)). (On the other hand, if we assume $\alpha \in C^{\infty}\left[-T_{0}, T_{1}\right]$, $\alpha^{\prime}(t) / \alpha(t)=b(t) \mid t^{\mid-\omega-1}$ for some $b \in C^{\infty}\left[-T_{0}, T_{1}\right], \pm b(0)>0$, then $\alpha$ is in the form (3.1.10) for some $B, \mu$.)

We may assume $b(t) \neq 0$ on $\left[-T_{0}, T_{1}\right]$.

We consider the operators of the following form in $U_{T_{1}}$.

$$
\begin{aligned}
& P(t, x ; \tau, \xi)=Q_{m}\left(t, x ; \tau, \xi^{\prime}, \alpha(t) \xi_{n}\right) \\
& \quad+\sum_{h=1}^{m}\left(\alpha^{\prime}(t) / \alpha(t)\right)^{h} Q_{m-h}\left(t, x ; \tau, \xi^{\prime}, \alpha(t) \xi_{n}\right),
\end{aligned}
$$

where $Q_{j}$ are the same as in (3.1.6).

By a technical reason, we impose stronger conditions on $Q_{m}$ than (3.1.7) ;

(i) the coefficients of $Q_{m}$ are real-valued,

(ii) $\tau_{0} \in C^{\infty}\left(U_{T_{1}}\right), \tau_{0}$ is independent of

$$
\begin{aligned}
& x^{\prime}=\left(x_{1}, \cdots, x_{n-1}\right), \text { and } \\
& Q_{m}\left(t, x ; \tau_{0}\left(t, x_{n}\right), 0,1\right)=0 \text { in } U_{T_{1}},
\end{aligned}
$$

(iii) $\left(\partial_{\tau} Q_{m}\right)\left(t, x ; \tau_{0}\left(t, x_{n}\right), 0,1\right) \neq 0$ in $U_{T_{1}}$. 
Theorem 3.6. Under the above situation, if (3.1.4) holds, then (3.1.8) holds with $C_{m, k}=2, k=1, \tau=\tau_{0}(0,0)$.

Remark 3.7. (a) Remark 3.4 (b) is also valid in this case.

(b) The condition that $P$ can be written in the form (3.1.11) is invariant under a coordinate transformation of the following form.

$$
\left\{\begin{array}{l}
s=t \\
y_{j}=f_{j}(x, t) \quad(j=1, \cdots, n-1) \\
y_{n}=f_{n}\left(x_{n}\right)+\alpha(t) b(0)^{-1}|t|^{\omega+1} h(t, x)=H(t, x),
\end{array}\right.
$$

where $f_{j}(0,0)=0(j=1, \cdots, n-1), f_{n}(0)=0$. Under this transformation, if we put the symbol of transformed operator as

$$
\begin{aligned}
\widetilde{P}= & \widetilde{Q}_{m}\left(s, y ; \sigma, \eta^{\prime}, \alpha(s) \eta_{n}\right) \\
& +\sum_{h=1}^{m}\left(\alpha^{\prime}(s) / \alpha(s)\right)^{h} \widetilde{Q}_{m-h}\left(s, y ; \sigma, \eta^{\prime}, \alpha(s) \eta_{n}\right),
\end{aligned}
$$

then (3.1.9) is also valid. Further, if $h(t, x)$ depends only on $\left(t, x_{n}\right)$, then

$$
\left\{\begin{array}{l}
\widetilde{Q}_{m}\left(s, y ; \sigma_{0}\left(s, y_{n}\right), 0,1\right)=0 \\
\left(\partial_{\sigma} \widetilde{Q}_{m}\right)\left(s, y ; \sigma_{0}\left(s, y_{n}\right), 0,1\right) \neq 0
\end{array}\right.
$$

where

$$
\begin{aligned}
\sigma_{0}\left(s, y_{n}\right)= & \left(\partial_{x_{n}} H\right)\left(s, x_{n}\left(s, y_{n}\right)\right) \tau_{0}\left(s, x_{n}\left(s, y_{n}\right)\right) \\
& -\left(\partial_{t} H\right)\left(s, x_{n}\left(s, y_{n}\right)\right) / \alpha(s),
\end{aligned}
$$

Note that the second term in the right hand side belongs to $C^{\infty}\left(U_{T_{1}}\right)$.

We give only a simple example. Many examples can be found in [20].

Example 3. 8. We consider

$$
P=D_{t}^{2}-e^{-2 / t} a(t, x) D_{x}^{2}+t^{-2} e^{-1 / t} b(t, x) D_{x},
$$

where $a, b \in C^{\infty}([0, T] \times \boldsymbol{R}), a(t, x)>0$ on $[0, T] \times \mathbb{R}$. For this operator, the C.P. is well-posed in $[0, T] \times \mathbb{R}$. ([15], [21], [6], [20], etc.) In this case, as in Example 3.5 (2), we get 


$$
\frac{|\operatorname{Im} b(0,0)|}{\sqrt{a(0,0)}} \leqq 2(q+1-p)+1,
$$

from $(3.1 .4)$.

\section{$\S 3.2$. Proof of Theorem 3.3}

By Remark 3.4 (c), we may assume $\hat{\tau}=0$. Further, we have only to consider the case where

$$
\operatorname{Im} \frac{Q_{m-1}(0,0 ; 0,0,1)}{\left(\partial_{r} Q_{m}\right)(0,0 ; 0,0,1)} \begin{cases}>0 & \text { (case (i)), } \\ <0 & \text { (case (ii)). }\end{cases}
$$

Let $\omega_{j}=3 \quad(j=0,1, \cdots, n-1), \omega_{n}=3 k+4$. And we perform the following coordinate transformation.

$$
\left\{\begin{array}{l}
t=s \rho^{-\omega_{0}} \\
x_{j}=y_{j} \rho^{-\omega_{j}}(j=1, \cdots, n) \quad(\rho>0) .
\end{array}\right.
$$

Then, $P$ is transformed into $P_{\rho}$ which is a partial differential operator on $B_{\left[-\widetilde{T}_{0}, \widetilde{T}_{1}\right]}$ for sufficiently large $\rho . \quad\left(B\right.$ is the open unit ball and $\left[-\widetilde{T}_{0}\right.$, $\left.\widetilde{T}_{1}\right]=[0,1]$ (resp. $[-1,0]$ ) in the case (i) (resp. (ii))). We have

$$
\begin{gathered}
P_{\rho, m-h}(s, y ; \sigma, \eta)=P_{m-h}\left(s \rho^{-\omega_{0}}, y \rho^{-\omega} ; \sigma \rho^{\omega_{0}}, \eta \rho^{\omega}\right) \\
=s^{-h} \rho^{\omega_{0} h} Q_{m-h}\left(s \rho^{-\omega_{0}}, y \rho^{-\omega} ; \sigma \rho^{\omega_{0}}, \eta^{\prime} \rho^{\omega^{\prime}}, \eta \eta s^{k} \rho^{\omega_{n}-k \omega_{0}}\right) \\
=s^{-h} \rho^{\omega_{0} h+\left(\omega_{n}-\omega_{0} k\right)(m-h)} s^{k(m-h)} \eta_{n}^{m-h} \\
\times Q_{m-h}\left(s \rho^{-\omega_{0}}, y \rho^{-\omega} ; \sigma s^{-k} \eta_{n}^{-1} \rho^{\omega_{0}+k \omega_{0}-\omega_{n}},\right. \\
\left.\eta^{\prime} s^{-k} \eta_{n}^{-1} \rho^{\omega^{\prime}+k \omega_{0}-\omega_{n}}, 1\right)
\end{gathered}
$$

where $\eta^{\prime}=\left(\eta_{1}, \cdots, \eta_{n-1}\right)$, etc.

We note that

$$
\left\{\begin{array}{l}
\omega_{0} h+\left(\omega_{n}-\omega_{0} k\right)(m-h)=4 m-h \\
\omega_{j}+\omega_{0} k-\omega_{n}=-1 \quad(j=0, \cdots, n-1), \\
Q_{m}(0,0 ; 0,0,1)=0 .
\end{array}\right.
$$

So, for any fixed positive integer $N$, we can write by Taylor expansion,

$$
\begin{aligned}
& P_{\rho}(s, y ; \sigma, \eta)=\rho^{4 m-1}\left[\left\{s ^ { k ( m - 1 ) } \eta _ { n } ^ { m - 1 } \left(\left(\partial_{\tau} Q_{m}\right)\left(0 ; 0, e_{n}\right) \sigma\right.\right.\right. \\
& \left.\left.\quad+\sum_{j=1}^{n-1}\left(\partial_{\xi_{j}} Q_{m}\right)\left(0 ; 0, e_{n}\right) \eta_{j}\right)+s^{k(m-1)-1} \eta_{n}^{m-1} Q_{m-1}\left(0 ; 0, e_{n}\right)\right\}
\end{aligned}
$$




$$
\left.+\sum_{j=1}^{N} \rho^{-j} A_{j}(s, y ; \sigma, \eta)+\rho^{-(N+1)} \tilde{A}_{N+1, \rho}(s, y ; \sigma, \eta)\right]
$$

where $e_{n}=(0, \cdots, 0,1) \in \mathbb{R}^{n}$ and

$$
\begin{aligned}
A_{j} \in \mathbb{F}=\left\{f(s, y ; \sigma, \eta)=\sum_{h=0}^{m} s^{-h} \sum_{k+|\alpha|=m-h} a_{k, \alpha}(s, y) \sigma^{k} \eta^{\alpha} ;\right. \\
\left.a_{k, \alpha} \in C^{\infty}\left(\mathbb{R}^{n+1}\right)\right\}, \\
\tilde{A}_{N+1, \rho} \in \widetilde{\mathbb{F}}=\left\{f_{\rho}(s, y ; \sigma, \eta)=\sum_{h=0}^{m} s^{-h} \sum_{k+|\alpha|=m-h} a_{k, \alpha}^{(o)}(s, y) \sigma^{k} \eta^{\alpha} ;\right. \\
\left.a_{k, \alpha}^{(\rho)} \text { is bounded in } C^{\infty}\left(B_{\left[-\tilde{T}_{0}, \widetilde{T}_{1}\right]}\right) \text { when } \rho \rightarrow+\infty\right\} .
\end{aligned}
$$

Further, $A_{1}$ does not include the term $\eta_{n}{ }^{m}$. So we have

$$
\begin{aligned}
e^{-i \rho y_{n}} \circ & P_{\rho} \circ e^{i \rho y_{n} \dagger}=\rho^{5 m-2}\left[\left\{s ^ { k ( m - 1 ) } \left(\left(\partial_{\tau} Q_{m}\right) D_{s}\right.\right.\right. \\
& \left.\left.+\sum_{j=1}^{n-1}\left(\partial_{\xi_{j}} Q_{m}\right) D_{y_{j}}\right)+s^{k(m-1)-1} Q_{m-1}\right\}+\sum_{j=1}^{N} \rho^{-j} B_{j}\left(s, y ; D_{s}, D_{y}\right) \\
& \left.+\rho^{-N-1} \widetilde{B}_{N+1, \rho}\left(s, y ; D_{s}, D_{y}\right)\right],
\end{aligned}
$$

where $B_{j}(s, y ; \sigma, \eta) \in \mathbb{F}(j=1, \cdots, N), \widetilde{B}_{N+1, \rho}(s, y ; \sigma, \eta) \in \widetilde{\mathbb{F}}$.

Now, for $\theta \in \mathbb{C}$.

$$
\begin{aligned}
& |s|^{-\theta} \circ e^{-i \rho y_{n} \circ} P_{\rho} \circ e^{i \rho y_{n}} \circ|s|^{\theta}=\rho^{5 m-2}\left[\left\{s ^ { k ( m - 1 ) } \left(\left(\partial_{\tau} Q_{m}\right) D_{s}\right.\right.\right. \\
& \left.\left.\quad+\sum_{j=1}^{n-1}\left(\partial_{\xi_{j}} Q_{m}\right) D_{y_{j}}\right)+s^{k(m-1)-1}\left(Q_{m-1}-i \theta\left(\partial_{\tau} Q_{m}\right)\right)\right\} \\
& \left.\quad+\sum_{j=1}^{N} \rho^{-j} E_{j}\left(s, y ; D_{s}, D_{y}\right)+\rho^{-N-1} \widetilde{E}_{N+1, \rho}\left(s, y ; D_{s}, D_{y}\right)\right],
\end{aligned}
$$

where $E_{j}(s, y ; \sigma, \eta) \in \mathbb{F} \quad(j=1, \cdots, N), \widetilde{E}_{N+1, \rho}(s, y ; \sigma, \eta) \in \widetilde{\mathbb{F}}$. We take $\theta$ as

$$
Q_{m-1}-i \theta\left(\partial_{\tau} Q_{m}\right)=0, \text { that is, } \theta=\frac{1}{i} \frac{Q_{m-1}}{\left(\partial_{\tau} Q_{m}\right)}
$$

Finally, we perform the linear coordinate transformation

$$
\left\{\begin{array}{l}
t=s \\
x_{j}=y_{j}-\frac{\left(\partial_{\xi_{j}} Q_{m}\right)}{\left(\partial_{\tau} Q_{m}\right)} \cdot s \quad(j=1, \cdots, n-1) \\
x_{n}=y_{n}
\end{array},\right.
$$

† $Q \circ R$ denotes the composition of operators $Q$ and $R$. A function is considered as a multiplication operator. 
then $P_{\rho}$ is transformed into $\widetilde{P}_{\rho}$, and

$$
\begin{aligned}
& |t|^{-\theta} \circ e^{-i \rho x_{n}} \circ \widetilde{P}_{\rho} \circ e^{i \rho x_{n}} \circ|t|^{\theta}=\rho^{5 m-2}\left\{t^{k(m-1)}\left(\partial_{\tau} Q_{m}\right) D_{t}\right. \\
& \left.\quad+\sum_{j=1}^{N} \rho^{-j} R_{j}\left(t, x ; D_{t}, D_{x}\right)+\rho^{-N-1} \widetilde{R}_{N+1, \rho}\left(t, x ; D_{t}, D_{x}\right)\right\},
\end{aligned}
$$

where $R_{j}(t, x ; \tau, \xi) \in \boldsymbol{F}(j=1, \cdots, N), \widetilde{R}_{N+1, \rho}(t, x ; \tau, \xi) \in \widetilde{\boldsymbol{F}}$. We put

$$
L_{\rho}=t^{k(m-1)}\left(\partial_{\tau} Q_{m}\right) D_{t}+\sum_{j=1}^{N} \rho^{-j} R_{j}+\rho^{-N-1} \widetilde{R}_{N+1, \rho} .
$$

To solve $L_{\rho} u=0$ asymptotically, we need the following lemma.

Lemma 3.9. Let $K$ be a compact set in $\boldsymbol{R}^{n}$. For any

$$
f(t, x)=\sum_{j=0}^{N} t^{-(N-j)(k+1)(m-1)-m}(\log |t|)^{j} f_{j}(t, x) \quad(N \geqq 0),
$$

where $f_{j} \in C_{K}^{\infty}=\left\{f \in C^{\infty}\left(\left[-\widetilde{T}_{0}, \widetilde{T}_{1}\right] \times \boldsymbol{R}^{n}\right)\right.$; supp $\left.f \subset\left[-\widetilde{T}_{0}, \widetilde{T}_{1}\right] \times K\right\} \quad(j=0$, $\cdots, N)$, a solution of $t^{k(m-1)} \partial_{t} u=f$ in $\left[-\widetilde{T}_{0}, \widetilde{T}_{1}\right] \times \boldsymbol{R}^{n}$ can be found in the form

$$
u(t, x)=\sum_{j=0}^{N+1} t^{-(N+1-j)(k+1)(m-1)}(\log |t|)^{j} g_{j}(t, x),
$$

where $g_{j} \in C_{K}^{\infty}(j=0,1, \cdots, N+1)$.

Proof. We have only to prove that for any $\tilde{f} \in C_{K}^{\infty}$ and any $l \geqq 1$, $j \geqq 0$, there exist $g_{h} \in C_{K}^{\infty}(h=0,1, \cdots, j+1)$ such that

$$
\begin{aligned}
& \int_{ \pm 1}^{t} \tau^{-l}(\log |\tau|)^{j} \tilde{f}(\tau, x) d \tau \\
& \quad=t^{-l+1} \sum_{h=0}^{j}(\log |t|)^{h} g_{h}(t, x)+(\log |t|)^{j+1} g_{j+1}(t, x) \\
& \quad\left(t \in\left[-\widetilde{T}_{0}, \widetilde{T}_{1}\right]\right) \quad(+ \text { in the case (i), - in the case (ii)). }
\end{aligned}
$$

By Taylor expansion, we can write

$$
\tilde{f}(t, x)=\sum_{\nu=0}^{l-1} t^{\nu} \tilde{f}_{\nu}(x)+t^{l} \tilde{\widetilde{f}}(t, x), \quad \text { where } \tilde{f}_{\nu} \in C_{0}^{\infty}(K), \tilde{\widetilde{f}} \in C_{K}^{\infty} .
$$

So,

(3.2.5) $\quad \int_{ \pm 1}^{t} \tau^{-\imath}(\log |\tau|)^{j} \tilde{f}(\tau, x) d \tau$ 


$$
=\sum_{\nu=0}^{l-1} \int_{ \pm 1}^{t} \tau^{\nu-l}(\log |\tau|)^{j} d \tau \tilde{f}_{\nu}(x)+\int_{ \pm 1}^{t}(\log |\tau|)^{j} \tilde{\widetilde{f}}(\tau, x) d \tau
$$

Now,

$$
\begin{aligned}
& \int_{ \pm 1}^{t} \tau^{\nu-l}(\log |\tau|)^{j} d \tau=\left[\frac{1}{\nu-l+1} \tau^{\nu-l+1}(\log |\tau|)^{j}\right]_{ \pm 1}^{t} \\
& \quad-\frac{j}{\nu-l+1} \int_{ \pm 1}^{t} \tau^{\nu-l}(\log |\tau|)^{j-1} d \tau, \text { if } 0 \leqq \nu \leqq l-2,
\end{aligned}
$$

and

$$
\int_{ \pm 1}^{t} \tau^{-1}(\log |\tau|)^{j} d \tau=\frac{1}{j+1}(\log |t|)^{j+1}
$$

Thus, the $\sum$-part in (3.2.5) is in the form (3.2.4). As for the last term of (3.2.5), we can write

$$
\int_{0}^{t} \tilde{\widetilde{f}}(\tau, x) d \tau=F(t, x)=t G(t, x), \text { where } F, G \in C_{K}^{\infty} .
$$

So,

$$
\begin{aligned}
& \int_{ \pm 1}^{t}(\log |\tau|)^{j} \tilde{\tilde{f}}(\tau, x) d \tau \\
& \quad=\left[(\log |\tau|)^{j} \tau G(\tau, x)\right]_{ \pm 1}^{t}-\int_{ \pm 1}^{t} j(\log |\tau|)^{j-1} G(\tau, x) d \tau .
\end{aligned}
$$

By induction, the last term of (3.2.5) is also in the form (3.2.4).

Q.E.D.

Now, we solve $L_{\rho}\left(\sum_{l=0}^{N} \rho^{-\imath} u_{l}(t, x)\right)=O\left(\rho^{-N-1}\right)$. That is,

$(3.2 .6 ; l) \quad t^{k(m-1)} D_{t} u_{l}=-\frac{1}{\partial_{\tau} Q_{m}} \sum_{j=1}^{l} R_{j}\left(u_{l-j}\right) \quad(l=0,1, \cdots, N)$.

First, $(3.2 .6 ; 0)$ is satisfied by any $u_{0}(t, x)=\varphi(x)$. We take $\varphi \in$ $C_{0}^{\infty}(K)$ and $\varphi(0)=1$. ( $K$ is a compact neighborhood of the origin in $\boldsymbol{R}^{n}$ which is chosen later.) By Lemma $3.9,(3.2 .6 ; l)$ is satisfied by

(3.2.7) $\quad u_{l}(t, x)=\sum_{j=0}^{l} t^{-(l-j)(k+1)(m-1)}\left(\log \mid t^{\mid}\right)^{j} g_{j}^{(l)}(t, x)$, where $g_{j}^{(l)} \in C_{K}^{\infty}(l=0,1, \cdots, N)$.

Let $\chi \in C^{\infty}(\mathbb{R})$ such that $\chi(t)=0(t \leqq 1 / 2), \chi(t)=1(t \geqq 1)$, and put 


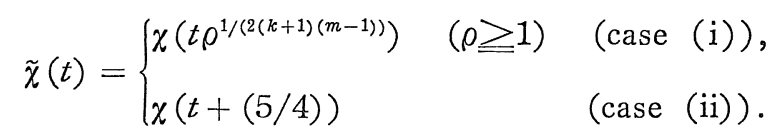

In the case (i), we have

$$
\begin{aligned}
\left(\partial_{t}^{\nu} \tilde{\chi}\right)(t) & =\rho^{\nu /(2(k+1)(m-1))}\left(\partial_{t}^{\nu} \chi\right)\left(t \rho^{1 /(2(k+1)(m-1))}\right), \\
& =t^{-\nu} \tilde{\chi}_{\nu}\left(t \rho^{1 /(2(k+1)(m-1))}\right)
\end{aligned}
$$

where supp $\tilde{\chi}_{\nu} \subset[1 / 2,1] \quad(\nu=1,2, \cdots)$.

Put

$$
\left\{\begin{array}{l}
V_{\rho}^{(N)}=\sum_{l=0}^{N} \rho^{-l} u_{l}(t, x) \\
U_{\rho}^{(N)}=e^{i \rho x_{l}}|t|^{\theta} \tilde{\chi}(t) V_{\rho}^{(N)} .
\end{array}\right.
$$

We define $\boldsymbol{B}_{l}^{(N)}, \mathbb{G}_{l}^{(N)}(l=0,1, \cdots, N+1 ; N=1,2, \cdots)$ as follows.

$$
\begin{aligned}
& \boldsymbol{B}_{l}^{(N)}=\left\{b_{\rho}(t, x)=\sum_{j ; \text { finite }} \psi_{j}\left(t \rho^{1 /(2(k+1)(n-1))}\right) h_{j}(t, x)\right. \\
& \left(\text { case (i)) or }=\sum_{j ; \text { finite }} \psi_{j}(t+(5 / 4)) h_{j}(t, x) \quad\right. \text { (case (ii)); } \\
& \left.\psi_{j} \in C_{0}^{\infty}([1 / 2,1]), h_{j} \in C_{K}^{\infty}\right\} \quad \text { when } l \leqq N, \\
& \boldsymbol{B}_{N+1}^{(N)}=\left\{b_{\rho}(t, x)=\sum_{j ; \text { finite }} \psi_{j}\left(t \rho^{1 /(2(k+1)(m-1))}\right) h_{\rho, j}(t, x)\right. \\
& \left(\text { case (i)) or }=\sum_{j ; \text { finite }} \psi_{j}(t+(5 / 4)) h_{\rho, j}(t, x)\right. \\
& \left(\text { case (ii)); } \psi_{j}=\chi \text { or } \in C_{0}^{\infty}([1 / 2,1]), h_{\rho, j}\right. \text { is bounded } \\
& \text { in } \left.C_{K}^{\infty} \text { when } \rho \rightarrow+\infty\right\} \quad \text { when } l=N+1, \\
& G_{l}^{(N)}=\left\{g_{\rho}(t, x)=\sum_{j=0}^{l} t^{-(l-1-j)(k+1)(m-1)-m}(\log |t|)^{j} b_{\rho, j}(t, x) ;\right. \\
& \left.b_{\rho, j} \in \boldsymbol{B}_{l}^{(N)}\right\} \quad(l=0,1, \cdots, N+1) .
\end{aligned}
$$

Then, we have

$$
\begin{aligned}
\widetilde{P}_{\rho}\left(U_{\rho}^{(N)}\right) & =\rho^{5 m-2}|t|^{\theta} e^{i \rho x_{n}} L_{\rho}\left(\tilde{\chi}(t) V_{\rho}^{(N)}(t, x)\right) \\
& =\rho^{5 m-2}|t|^{\theta} e^{i \rho x_{n}} \sum_{l=0}^{N+1} \rho^{-l} G_{l, \rho}^{(N)}(t, x),
\end{aligned}
$$

where $G_{l, o}^{(N)} \in \mathcal{G}_{l}^{(N)} \quad(l=0,1, \cdots, N+1)$.

Now, we have the following estimate for $\widetilde{P}_{\rho}$ from (3.1.4) by Lemma 2. 10 in Section 2.3. 
Lemma 3. 10. There exists a constant $C$ such that for sufficiently large $\rho$, the following estimates hold.

$$
\begin{aligned}
& \|\widetilde{u}\|_{p, t}^{(4)} \leqq C \rho^{(q-p)(3 k+4)}\left\|\widetilde{P}_{\rho} \widetilde{u}\right\|_{q, t}^{(3)} \\
& \text { for any } t \in\left[-\widetilde{T}_{0}, \widetilde{T}_{1}\right], \widetilde{u} \in C_{0}^{\infty}\left(B_{\widetilde{T}_{1}}\right) \text {, where } \\
& \|v\|_{q, t}^{(3)}= \begin{cases}\|v\|_{H^{q}\left(B_{t}\right)} & (q \geqq 0) \\
\sup _{w \in C_{0}^{\infty}\left(B_{t}\right)} \frac{\left|(v, w)_{L^{2}\left(B_{t}\right)}\right|}{\left\|D_{x_{n}}^{-q} w\right\|_{L^{2}\left(B_{t}\right)}} & (q \leqq 0),\end{cases} \\
& \|v\|_{p, t}^{(4)}= \begin{cases}\left\|D_{x_{n}}^{p} v\right\|_{L^{2}\left(B_{t}\right)} & (p \geq 0) \\
\|v\|_{H^{p}\left(B_{t}\right)} & (p \leqq 0),\end{cases} \\
& B_{t}=B_{\left[-\widetilde{T}_{0}, t\right]}\left(t \in\left[-\widetilde{T}_{0}, \widetilde{T}_{1}\right]\right) .
\end{aligned}
$$

Now, we fix $0<t_{0} \leqq 1 / 2$ and a compact neighborhood $K$ of the origin in $\mathbb{R}^{n}$ such that $\left[0, \frac{3}{4}\right] \times K \subset B$. And we take

$$
t_{\rho}= \begin{cases}t_{0} & (\operatorname{case}(\mathrm{i})) \\ -t_{0} \rho^{-1 /(2(k+1)(m-1))} & (\operatorname{case}(\mathrm{ii}))\end{cases}
$$

Then, we can estimate $U_{\rho}^{(N)}, \widetilde{P}_{\rho} U_{\rho}^{(N)}$ as follows.

Lemma 3. 11 . There exist positive constants $\delta$ and $C$ such that for sufficiently large $\rho$ and $N$, there hold the following estimates.

$$
\left\|U_{\rho}^{(N)}\right\|_{p, t_{\rho}}^{(4)} \geqq \begin{cases}\delta \cdot \rho^{p} & (\operatorname{case}(\mathrm{i})) \\ \delta \cdot \rho^{p-(2 \operatorname{Re} \theta+1) /(4(k+1)(m-1))} & (\operatorname{case}(\mathrm{ii})) .\end{cases}
$$

(3.2.11) $\quad\left\|\widetilde{P}_{\rho} U_{\rho}^{(N)}\right\|_{q, t_{\rho}}^{(3)}$

$$
\leqq \begin{cases}C \cdot \rho^{5 m-(5 / 2)-\operatorname{Re} \theta /(2(k+1)(m-1))+q+(2 m-1) /(4(k+1)(m-1))} & (\text { case }(\mathrm{i})) \\ C \cdot \rho^{5 m-2+q} & \text { (case (ii)). }\end{cases}
$$

Note that we are assuming that $\operatorname{Re} \theta>0$ in the case (i) and $\operatorname{Re} \theta<0$ in the case (ii).

Proof of (3.2.10).

(1) Case (i), $p \geqq 0$. By $\iota_{0}(t, x)=\varphi(x) \in C_{0}^{\infty}(K)$ and $\varphi(0)=1$, we have 


$$
\left\|e^{i \rho x_{n}} t^{\theta} \tilde{\chi}(t) u_{0}(t, x)\right\|_{p, t_{0}}^{(4)} \geqq \tilde{\delta} \cdot \rho^{p} \quad(\tilde{\delta}>0) .
$$

On the other hand,

$$
\tilde{\chi}(t)=0 \quad \text { if } \quad 0 \leqq t \leqq 2^{-1} \rho^{-1 /(2(k+1)(m-1))},
$$

so we can estimate $t^{-1}$ by $2 \rho^{1 /(2(k+1)(m-1))}$. Therefore,

$$
\left\|e^{i \rho x_{n}} t^{\theta} \tilde{\chi}(t) u_{l}(t, x)\right\|_{p, t_{0}}^{(4)} \leqq C \cdot \rho^{p+(l / 2)} \quad(l=1, \cdots, N) .
$$

So, we get the result.

(2) Case (i), $p \leqq 0$. By definition, we have

$$
\text { (3. 2.12) } \quad\left\|U_{\rho}^{(N)}\right\|_{p, t_{\rho}}^{(4)}=\sup _{w \in C_{0}^{\infty}\left(B_{t_{\rho}}\right)} \frac{\left|\left(U_{\rho}^{(N)}, w\right)_{L^{2}\left(B_{t_{\rho}}\right)}\right|}{\|w\|_{H^{-p}\left(B_{t_{\rho}}\right)}} .
$$

If we take $w=e^{i \rho x_{n}} \cdot v, v \in C_{0}^{\infty}\left(B_{t_{0}}\right)$, we have

$$
\|w\|_{H^{-p}\left(B_{t_{0}}\right)} \leqq \rho^{-p}\|v\|_{H^{-p}\left(B_{t_{0}}\right)} \quad(\rho \geqq 1) .
$$

So,

$$
\left\|U_{\rho}^{(N)}\right\|_{p, t_{0}}^{(4)} \geqq \sup _{v \in C_{0}^{\infty}\left(B_{t_{0}}\right)} \frac{\left.\mid\left(t^{\theta} \tilde{\chi}(t) V_{\rho}^{(N)}(t, x), v(t, x)\right)_{L^{2}\left(B_{t} 0\right.}\right) \mid}{\|v\|_{H^{-p}\left(B_{t_{0}}\right)}} \cdot \rho^{p}
$$

For suitable $v$, we have

$$
\begin{aligned}
& \left|\left(t^{\theta} \tilde{\chi}(t) u_{0}(t, x), v(t, x)\right)_{L^{2}\left(B_{t_{0}}\right)}\right| \geqq \tilde{\delta}>0, \\
& \left|\left(t^{\theta} \tilde{\chi}(t) u_{l}, v\right)_{L^{2}\left(B_{t_{0}}\right)}\right| \leqq C \rho^{l / 2} \quad(l=1, \cdots, N) .
\end{aligned}
$$

So, we have

$$
\left\|U_{\rho}^{(N)}\right\|_{p, t_{0}}^{(4)} \geq \delta \cdot \rho^{p} \quad(\delta>0) .
$$

(3) Case (ii), $p \geqq 0$. In $\left\{-2 t_{0} \rho^{-1 /(2(k+1)(m-1))} \leqq t \leqq-t_{0} \rho^{-1 /(2(k+1)(m-1))}\right\}$, we have

$$
\left.|| t\right|^{\theta} \mid \geqq \tilde{\delta} \cdot \rho^{-\operatorname{Re} \theta /(2(k+1)(m-1))} \quad(\tilde{\delta}>0)
$$

So,

$$
\begin{aligned}
& \left\|e^{i \rho x_{n}}|t|^{\theta} \tilde{\chi}(t) u_{0}(t, x)\right\|_{p, t_{\rho}}^{(4)} \\
& \quad \geq \delta \cdot \rho^{p-\operatorname{Re} \theta /(2(k+1)(m-1))-1 /(4(k+1)(m-1))} \quad(\delta>0) .
\end{aligned}
$$

On the other hand,

$$
\left\|e^{i \rho x_{n}}|t|^{\theta} \tilde{\chi} u_{l}\right\|_{p, t_{\rho}}^{(4)} \leqq C \rho^{p-\operatorname{Re} \theta /(2(k+1)(m-1))+l / 2} \quad(l=1, \cdots, N) .
$$

So, we get the result. 
(4) Case (ii), $p \leqq 0$. In (3.2.12), if we take $w=e^{i \rho x_{n}} v\left(t \rho^{1 /(2(k+1)(m-1))}\right.$, $x), v \in C_{0}^{\infty}\left(\left(-2 t_{0},-t_{0}\right) \times K\right)$, then

$$
\|w\|_{H^{-p}\left(B_{t}\right)} \leqq \rho^{-p-1 /(4(k+1)(m-1))}\|v\|_{H^{-p}\left(\left(-2 t_{0},-t_{0}\right) \times K\right)} .
$$

So, as above,

$$
\begin{aligned}
& \left\|U_{\rho}^{(N)}\right\|_{p, t_{\rho}}^{(4)} \\
& \geq \sup _{v \in C_{0}^{\infty}\left(\left(-2 t_{0},-t_{0}\right) \times K\right)} \frac{\left|\left(\left.|t|\right|^{\theta} \tilde{\chi} V_{\rho}^{(N)}, v\left(t \rho^{1 /(2(k+1)(m-1))}, x\right)\right)_{L^{2}\left(B_{t}\right)}\right|}{\|v\|_{H^{-p}\left(\left(-2 t_{0},-t_{0}\right) \times K\right)}} \\
& \quad \times \rho^{p+1 /(4(k+1)(m-1))} \\
& \geq \delta \cdot \rho^{p+1 /(4(k+1)(m-1))-\operatorname{Re} \theta /(2(k+1)(m-1))-1 /(2(k+1)(m-1))} \quad(\delta>0) .
\end{aligned}
$$

Proof of (3.2.11).

(1) Case (i), $q \geqq 0$. In (3.2.8), $\quad \widetilde{P}_{\rho}\left(U_{\rho}^{(N)}\right)=0$ when $t \leqq$ $2^{-1} \rho^{-1 /(2(k+1)(m-1))}$. And, $G_{l, \rho}^{(N)}=0(l \leqq N)$, when $\rho^{-1 /(2(k+1)(m-1))} \leqq t \leqq t_{0}$, so, in this interval, we have

$$
\begin{aligned}
& \left\|\widetilde{P}_{\rho} U_{\rho}^{(N)}\right\|_{H^{q}} \leqq C \rho^{5 m-2-N-1+q+(N(k+1)(m-1)+m) /(2(k+1)(m-1))} \\
& \quad \leqq C \rho^{5 m-3+q-(N / 2)+(m / 4)} . \\
& \operatorname{In}\left\{\frac{1}{2} \rho^{-1 /(2(k+1)(m-1))} \leqq t \leqq \rho^{-1 /(2(k+1)(m-1))}\right\}, \text { we have } \\
& \left\|t^{\theta} e^{i \rho x_{n}} G_{l, \rho}^{(N)}\right\|_{H^{q}} \\
& \leqq C \rho^{-\operatorname{Re} \theta /(2(k+1)(m-1))+q+((l-1)(k+1)(m-1)+m) /(2(k+1)(m-1))-1 /(4(k+1)(m-1))} .
\end{aligned}
$$

So, in this interval,

$$
\left\|\widetilde{P}_{\rho} U_{\rho}^{(N)}\right\|_{H^{q}} \leqq C \rho^{5 m-2-\operatorname{Re} \theta /(2(k+1)(m-1))+q-(1 / 2)+(2 m-1) /(4(k+1)(m-1))} .
$$

(2) Case (ii), $q \geqq 0$. In $\left\{-1 / 4 \leqq t \leqq t_{\rho}\right\}, G_{l, \rho}^{(N)}=0 \quad(l \leqq N)$. So, in this interval, we have

$$
\begin{aligned}
\left\|\widetilde{P}_{\rho} U_{\rho}^{(N)}\right\|_{H^{q}} & \leqq C \rho^{5 m-2-N-1+q+(N(k+1)(m-1)+m) /(2(k+1)(m-1))-\operatorname{Re} \theta /(2(k+1)(m-1))} \\
& \leqq C \rho^{5 m-3+q-(N / 2)+m-\operatorname{Re} \theta /(2(k+1)(m-1))} .
\end{aligned}
$$

In $\{-1 \leqq t \leqq-1 / 4\}$, we have

$$
\left\|\widetilde{P}_{\rho} U_{\rho}^{(N)}\right\|_{H^{q}} \leqq C \rho^{5 m-2+q} .
$$


Therefore we have the result.

(3) When $q \leqq 0$. We solve

$$
D_{x_{n}}^{|q|} W_{\rho}^{(N)}-\widetilde{P}_{\rho} U_{\rho}^{(N)}=O\left(\rho^{-N-1+5 m-2}\right) .
$$

We put

$$
W_{\rho}^{(N)}=\rho^{5 m-2-|q|}|t|^{\theta} e^{i \rho x_{n}} \sum_{l=0}^{N} \rho^{-l} H_{l, \rho}(t, x),
$$

then,

$$
\begin{aligned}
D_{x_{n}}^{|q|} W_{\rho}^{(N)}= & \rho^{5 m-2}|t|^{\theta} e^{i \rho x_{n}} \sum_{l=0}^{N+|q|} \rho^{-l}\left(H_{l, \rho}+D_{x_{n}} H_{l-1, \rho}+\cdots\right. \\
& \left.+D_{x_{n}}^{|q|} H_{l-|q|, \rho}\right), \quad\left(H_{-1, \rho}=\cdots=H_{-|q|, \rho}=0\right) .
\end{aligned}
$$

So, (3.2.13) can be solved by

$$
H_{l, \rho}=G_{l, \rho}^{(N)}-\sum_{j=1}^{|q|} D_{x_{n}}^{j} H_{l-j, \rho} \quad(l=0,1, \cdots, N) .
$$

If we take $H_{l, \rho}$ as these, we have $H_{l, \rho} \in \boldsymbol{G}_{l}^{(N)}$. Further, we have

$$
\widetilde{P}_{\rho} U_{\rho}^{(N)}-D_{x_{n}}^{|q|} W_{\rho}^{(N)}=\rho^{-N-1+5 m-2}|t|^{\theta} e^{i \rho x_{n}} \tilde{H}_{\rho}^{(N)},
$$

where $\tilde{H}_{\rho}^{(N)} \in \boldsymbol{G}_{N+1}^{(N)}$.

(3-1) Case (i). As in the case $q \geqq 0$, we have

$$
\left\|W_{\rho}^{(N)}\right\|_{L^{2}\left(B_{t_{0}}\right)} \leqq C \rho^{5 m-2-|q|-\operatorname{Re} \theta /(2(k+1)(m-1))-(1 / 2)+(2 m-1) /(4(k+1)(m-1))} .
$$

On the other hand, from the energy inequality for $D_{x_{n}}^{|q|}$,

$$
\|w\|_{L^{2}\left(B_{t_{0}}\right)} \leqq C\left\|D_{x_{n}}^{|q|} w\right\|_{L^{2}\left(B_{t_{0}}\right)} \quad \text { for any } w \in C_{0}^{\infty}\left(B_{t_{0}}\right) \text {, }
$$

we have

$$
\|v\|_{-|q|, t_{0}}^{(3)} \leqq C\|v\|_{L^{2}\left(B_{t_{0}}\right)} .
$$

So, we have

$$
\left\|\widetilde{P}_{\rho} U_{\rho}^{(N)}-D_{x_{n}}^{|q|} W_{\rho}^{(N)}\right\|_{q, t_{0}}^{(3)} \leqq C \rho^{-N-1+5 m-2+(N(k+1)(m-1)+m) /(2(k+1)(m-1))} .
$$

Now,

$$
\begin{aligned}
\left\|D_{x_{n}}^{|q|} W_{\rho}^{(N)}\right\|_{q, t_{0}}^{(3)}= & \sup _{w \in C_{0}^{\infty}\left(B_{0}\right)} \frac{\left|\left(D_{x_{n}}^{|q|} W_{\rho}^{(N)}, w\right)_{L^{2}\left(B_{t_{0}}\right)}\right|}{\left\|D_{x_{n}}^{|q|} w\right\|_{L^{2}\left(B_{t_{0}}\right)}} \\
& \leqq\left\|W_{\rho}^{(N)}\right\|_{L^{2}\left(B_{t_{0}}\right)}
\end{aligned}
$$

So, we have 


$$
\left\|\widetilde{P}_{\rho} U_{\rho}^{(N)}\right\|_{q, t_{0}}^{(3)} \leqq C \rho^{5 m-(5 / 2)+q-\operatorname{Re} \theta /(2(k+1)(m-1))+(2 m-1) /(4(k+1)(m-1))} .
$$

(3-2) Case (ii). As in the case $q \geqq 0$, we have

$$
\left\|W_{\rho}^{(N)}\right\|_{L^{2}\left(B_{t_{\rho}}\right)} \leqq C \rho^{5 m-2-|q|} .
$$

And, as in the case (i), we have

$$
\begin{aligned}
& \left\|\widetilde{P}_{\rho} U_{\rho}^{(N)}-D_{x_{n}}^{|q|} W_{\rho}^{(N)}\right\|_{q, t_{\rho}}^{(3)} \\
& \leqq C \rho^{-N-1+5 m-2+(N(k+1)(m-1)+m) /(2(k+1)(m-1))-\operatorname{Re} \theta /(2(k+1)(m-1))}, \\
& \left\|D_{x_{n}}^{|q|} W_{\rho}^{(N)}\right\|_{q, t_{\rho}}^{(3)} \leqq\left\|W_{\rho}^{(N)}\right\|_{L^{2}\left(B_{\rho}\right)} .
\end{aligned}
$$

So, we have the result.

Q.E.D.

From Lemma 3.10, 3.11, we have

$$
\begin{gathered}
p \leqq(q-p)(3 k+4)+5 m-\frac{5}{2}-\frac{\operatorname{Re} \theta}{2(k+1)(m-1)}+q \\
\quad+\frac{2 m-1}{4(k+1)(m-1)} \quad(\text { case (i)), } \\
p-\frac{1}{2(k+1)(m-1)}-\frac{\operatorname{Re} \theta}{4(k+1)(m-1)} \\
\leqq(q-p)(3 k+4)+5 m-2+q \quad \text { (case (ii)). }
\end{gathered}
$$

So,

$$
\begin{aligned}
\pm \operatorname{Re} \theta \leqq 6(k+1)(k+2)(m-1)(q+m-1-p) \\
(+ \text { in the case (i), }- \text { in the case (ii)). }
\end{aligned}
$$

If we put

$$
C_{m, k}=6(k+1)(k+2)(m-1),
$$

we get (3.1.8).

Q.E.D.

\section{$\S 3.3 . \quad$ Proof of Theorem 3.6}

We can solve

$$
\left\{\begin{array}{l}
\left(\partial_{t} H\right)\left(t, x_{n}\right)=\alpha^{\prime}(t) \tau_{0}\left(t, x_{n}\right)\left(\partial_{x_{n}} H\right)\left(t, x_{n}\right) \\
H\left(0, x_{n}\right)=x_{n}
\end{array}\right.
$$


in a neighborhood of the origin in $U_{T_{1}}$. And by Lemma in Appendix II, this solution $H$ can be written as

$$
H\left(t, x_{n}\right)=x_{n}+\alpha(t)|t|^{\omega+1} \tilde{H}\left(t, x_{n}\right), \tilde{H} \in C^{\infty} .
$$

So, by Remark 3.7 , we may assume $\tau_{0} \equiv 0$. Further, we have only to consider the case where

$$
\operatorname{Im} \frac{Q_{m-1}(0,0 ; 0,0,1)}{\left(\partial_{\tau} Q_{m}\right)(0,0 ; 0,0,1)} \begin{cases}>0 & \text { (case (i)) } \\ <0 & \text { (case (ii)). }\end{cases}
$$

The essential idea is the same as in the proof of Theorem 3.3. But, we can not perform a sympletic dilation like (3.2.2). So, we skip to the next step and this is the reason why we need (3.1.12).

First, we have

$$
e^{-i \rho x_{n} \circ P \circ e^{i \rho x_{n}}}=P\left(t, x ; D_{t}, D_{x^{\prime}}, \rho+D_{x_{l l}}\right)
$$

where

$$
\begin{aligned}
& P_{m-h}\left(t, x ; \tau, \xi^{\prime}, \rho+\xi_{n}\right) \\
& =\left(\alpha^{\prime}(t) / \alpha(t)\right)^{h} Q_{m-h}\left(t, x ; \tau, \xi^{\prime}, \alpha(t)\left(\rho+\xi_{n}\right)\right) \\
& =\left(\alpha^{\prime}(t) / \alpha(t)\right)^{h} \alpha(t)^{m-h} \rho^{m-h} \\
& \quad \times Q_{m-h}\left(t, x ; \frac{\tau}{\alpha(t) \rho}, \frac{\xi^{\prime}}{\alpha(t) \rho}, 1+\frac{\xi_{n}}{\rho}\right) .
\end{aligned}
$$

Now, we have

$$
Q_{m}\left(t, x ; 0,0, \xi_{n}\right)=0 \text { for any }\left(t, x ; \xi_{n}\right) .
$$

So, by Taylor expansion w.r.t. $(\tau, \xi)$, we have

$$
\begin{aligned}
& P\left(t, x ; \tau, \xi^{\prime}, \rho+\xi_{n}\right)=\rho^{m-1}\left\{\alpha ( t ) ^ { m - 1 } \left(\left(\partial_{\tau} Q_{m}\right)\left(t, x ; 0, e_{n}\right) \tau\right.\right. \\
& \left.\quad+\sum_{j=1}^{n-1}\left(\partial_{\xi_{j}} Q_{m}\right)\left(t, x ; 0, e_{n}\right) \xi_{j}\right)+\alpha^{\prime}(t) \alpha(t)^{m-2} Q_{m-1}\left(t, x ; 0, e_{n}\right) \\
& \left.\quad+\sum_{l=1}^{m-1} \rho^{-l} \alpha(t)^{m-l-1}\left(\alpha^{\prime}(t) / \alpha(t)\right)^{l+1} S_{l}(t, x ; \tau, \xi)\right\},
\end{aligned}
$$

where $S_{l}$ is a polynomial of degree $(l+1)$ w.r.t. $(\tau, \xi)$ with $C^{\infty}$-coefficients (note that $\alpha(t) / \alpha^{\prime}(t) \in C^{\infty}\left[-T_{0}, T_{1}\right]$ ), and $e_{n}=(0, \cdots, 0,1) \in \boldsymbol{R}^{n}$.

Next, we solve 


$$
\left\{\begin{array}{l}
\left(\partial_{\tau} Q_{m}\right)\left(t, x ; 0, e_{n}\right) \partial_{t} f_{h}+\sum_{j=1}^{n-1}\left(\partial_{\xi_{j}} Q_{m}\right)\left(t, x ; 0, e_{n}\right) \partial_{x_{j}} f_{h}=0 \\
f_{h}(0, x)=x_{h} \quad(h=1, \cdots, n-1) .
\end{array}\right.
$$

This can be solved in a neighborhood of the origin in $U_{T_{1}}$, and

$$
\left\{\begin{array}{l}
s=t \\
y_{j}=f_{j}(x, t) \quad(j=1, \cdots, n-1) \\
y_{n}=x_{n}
\end{array}\right.
$$

is a coordinate transformation in a neighborhood of the origin in $U_{T_{1}}$. We put

$$
\left\{\begin{array}{l}
a_{0}(s, y)=\left(\partial_{\tau} Q_{m}\right)(s, x(s, y) ; 0,0,1) \\
S_{0}(s, y)=\frac{Q_{m-1}(s, x(s, y) ; 0,0,1)}{a_{0}(s, y)} .
\end{array}\right.
$$

Under the above transformation, if we write the transformed operators as $\widetilde{P}$, $\widetilde{S}_{l}$, then $e^{-i \rho x_{n}} \circ P \circ e^{i \rho x_{n}}$ is transformed into

$$
\begin{gathered}
e^{-i \rho y_{n}} \circ \widetilde{P} \circ e^{i \rho y_{n}}=\rho^{m-1}\left\{\alpha(s)^{m-2} a_{0}(s, y)\left(\alpha(s) D_{s}+S_{0}(s, y) \alpha^{\prime}(s)\right)\right. \\
\left.+\sum_{l=1}^{m-1} \rho^{-l} \alpha(s)^{m-l-1}\left(\alpha^{\prime}(s) / \alpha(s)\right)^{l+1} \widetilde{S}_{l}\left(s, y ; D_{s}, D_{y}\right)\right\} .
\end{gathered}
$$

We may assume that this operator is defined on $U_{T_{1}}$.

Hereafter, we will write $(t, x)$ instead of $(s, y)$.

We take

$$
\beta(t, x)=-i \int_{ \pm 1}^{t} \frac{\alpha^{\prime}(u)}{\alpha(u)} S_{0}(u, x) d u=-i \int_{ \pm 1}^{t} \frac{b(u) S_{0}(u, x)}{|u|^{\omega+1}} d u .
$$

By Taylor expansion, we can write

$$
b(u) S_{0}(u, x)=\sum_{j=0}^{\omega} u^{j} e_{j}(x)+u^{\omega+1} e(u, x) .
$$

So, we have

$$
\beta(t, x)=c(t, x)|t|^{-\omega}+\mu(x) \log |t|,
$$

where $c \in C^{\infty}\left(U_{T_{1}}\right), \mu \in C^{\infty}\left(U_{x}\right) \quad\left(U_{x}=\left\{x \in \mathbb{R}^{n} ;(0, x) \in U\right\}\right)$, and $c(0, x)$ $= \pm \frac{i}{\omega} b(0) S_{0}(0, x)=i B(0) S_{0}(0, x) \quad(+$ in the case (i), - in the case (ii)). And, we have

$$
e^{-\beta(t, x)} \circ\left(\alpha(t) D_{t}+\alpha^{\prime}(t) S_{0}(t, x)\right) \circ e^{\beta(t, x)}=\alpha(t) D_{t},
$$




$$
e^{-\beta(t, x)} \circ \widetilde{S}_{l} \circ e^{\beta(t, x)}=|t|^{-(l+1)(\omega+1)}\left(H_{l}+(\log |t|) \widetilde{H}_{l}\right),
$$

where $H_{l}, \widetilde{H}_{l}$ are differential operators of order $(l+1)$ with $C^{\infty}$-coefficients. Therefore, if we fix a positive number $\delta$, we have

$$
\begin{aligned}
& e^{-\beta(t, x)} \circ e^{-i \rho x_{n}} \circ \widetilde{P}_{0} e^{i \rho x_{n}} \circ e^{\beta(t, x)} \\
& =\rho^{m-1}\left\{\alpha(t)^{m-1} a_{0}(t, x) D_{t}+\sum_{l=1}^{m-1} \rho^{-l} \alpha(t)^{m-l-1-\delta} A_{l}\right\},
\end{aligned}
$$

where $A_{l}$ is a differential operator of order $(l+1)$ with $C^{\infty}$-coefficients. We need the following lemma instead of Lemma 3.9.

Lemma 3. 12. Let $f \in C^{\infty}\left(\left[-T_{0}, T_{1}\right] \times \boldsymbol{R}^{n}\right)$, supp $f \subset\left[-T_{0}, T_{1}\right]$ $\times K, \nu>0, \delta>0$ ( $K$ is a compact subset of $\boldsymbol{R}^{n}$ ). Then, a solution of

$$
D_{t} u=\alpha(t)^{-\nu} f
$$

can be found in the form

$$
u(t, x)=\alpha(t)^{-\nu-\delta} g(t, x),
$$

where $g \in C^{\infty}\left(\left[-T_{0}, T_{1}\right] \times \boldsymbol{R}^{n}\right)$, supp $g \subset\left[-T_{0}, T_{1}\right] \times K$.

Proof. We have only to consider

$$
\begin{gathered}
G(t, x)=\alpha(t)^{\nu} \int_{ \pm 1}^{t} \alpha(u)^{-\nu} f(u, x) d u=\int_{ \pm 1}^{t}\left(\frac{\alpha(t)}{\alpha(u)}\right)^{\nu} f(u, x) d u \\
(+ \text { in the case (i), - in the case (ii)). }
\end{gathered}
$$

There holds

$$
0 \leqq \frac{\alpha(t)}{\alpha(u)} \leqq 1 \quad \text { for } \quad 0 \leqq|t| \leqq|u| \leqq 1
$$

So, $G \in C^{\infty}\left(\left(\left[-T_{0}, T_{1}\right]-\{0\}\right) \times \boldsymbol{R}^{n}\right)$ and bounded when $t \rightarrow 0$. Further,

$$
\partial_{t} G=\nu \frac{b(t)}{|t|^{\omega+1}} G+f(t, x),
$$

and by induction,

$$
\partial_{t}^{j} G=|t|^{-j(\omega+1)}\left(a_{j}(t, x) G(t, x)+b_{j}(t, x)\right),
$$

where $a_{j}, b_{j} \in C^{\infty}\left(\left[-T_{0}, T_{1}\right] \times \boldsymbol{R}^{n}\right), \operatorname{supp} a_{j}$, supp $b_{j} \subset\left[-T_{0}, T_{1}\right] \times K$. So, $\alpha(t)^{\delta} G(t, x) \in C^{\infty}\left(\left[-T_{0}, T_{1}\right] \times \boldsymbol{R}^{n}\right)$. Q.E.D. 
(As a matter of fact, we can prove that $u$ can be taken in the form $u=\alpha(t)^{-\nu} \mid t_{\mid}^{\omega+1} \widetilde{g}(t, x)$, where $\widetilde{g} \in C^{\infty}\left(\left[-T_{0}, T_{1}\right] \times \mathbb{R}^{n}\right)$, supp $\widetilde{g} \subset\left[-T_{0}, T_{1}\right]$ $\times K$, by the method in Appendix II.)

We put

$$
L_{\rho}=\alpha(t)^{m-1} a_{0}(t, x) D_{t}+\sum_{l=1}^{m-1} \rho^{-l} \alpha(t)^{m-l-1-\delta} A_{l},
$$

and we will solve

$$
L_{\rho}\left(\sum_{l=0}^{N} \rho^{-l} u_{l}\right)=O\left(\rho^{-N-1}\right) .
$$

That, is,

$$
\begin{gathered}
(3.3 .1 ; l) \quad \alpha(t)^{m-1} D_{t} u_{l}=-\frac{1}{a_{0}(t, x)} \sum_{j=1}^{m-1} \alpha(t)^{m-j-1-\delta} A_{j}\left(u_{l-j}\right) \\
\left(u_{-1}=\cdots=u_{-m+1}=0\right), \quad l=0,1, \cdots, N .
\end{gathered}
$$

First, $(3.3 .1 ; 0)$ is satisfied by any $u_{0}(t, x)=\varphi(x) \in C_{0}^{\infty}\left(\mathbb{R}^{n}\right)$. Now, we fix $\delta$ which satisfies $0<\delta<\left|\operatorname{Im} S_{0}(0,0)\right| / 2$, and we take a neighbor$\operatorname{hood} \tilde{U}$ of the origin so small that

$$
|\nu(t, x)-\nu(0,0)| \leqq \delta \quad \text { on } \quad \widetilde{U}_{\left[-T_{0}, T_{1}\right]},
$$

where $\nu(t, x)=-\frac{c(t, x)}{B(t)} \in C^{\infty}\left(U_{T_{1}}\right)$. Further we take $\widetilde{T} \in(0, T]$ and a compact neighborhood $K$ of the origin in $\mathbb{R}^{n}$ such that $\left[-\widetilde{T}_{0}, \widetilde{T}_{1}\right]$ $\times K \subset \widetilde{U}$, where $\left[-\widetilde{T}_{0}, \widetilde{T}_{1}\right]=[0, \widetilde{T}]$ (resp. $[-\widetilde{T}, 0]$ ) in the case (i) (resp. (ii)), and we take $\varphi \in C_{0}^{\infty}(K), \varphi(0)=1$. Then, (3.3.1) is solved by means of Lemma 3.12 in the form

$$
u_{l}(t, x)=\alpha(t)^{-l(1+2 \delta)} g_{l}(t, x),
$$

where $g_{l} \in C_{K}^{\infty}=\left\{f \in C^{\infty}\left(\left[-\widetilde{T}_{0}, \widetilde{T}_{1}\right] \times \mathbb{R}^{n}\right)\right.$; supp $\left.f \subset\left[-\widetilde{T}_{0}, \widetilde{T}_{1}\right] \times K\right\}$.

Let $\chi \in C^{\infty}(\boldsymbol{R})$ be as in the proof of Theorem 3.3, and put

$$
\tilde{\chi}(t)= \begin{cases}\chi\left(\alpha(t) \rho^{1 /(2(1+2 \delta))}\right) & (\text { case }(\mathrm{i})) \\ \chi\left(\frac{t}{\widetilde{T}}+\frac{3}{2}\right) & (\text { case (ii)). }\end{cases}
$$

In the case (i), we have

$$
\partial_{t}^{v} \tilde{\chi}(t)=|t|^{-\nu(\omega+1)} \sum_{h=1}^{N_{\nu}} \chi_{h}^{(\nu)}\left(\alpha(t) \rho^{1 /(2(1+2 \delta))}\right) d_{h}^{(\nu)}(t),
$$


where $\chi_{h}^{(\nu)} \in C_{0}^{\infty}([1 / 2,1]), d_{h}^{(\nu)} \in C^{\infty}(\boldsymbol{R}) \quad(\nu=1,2, \cdots)$.

Put

$$
\left\{\begin{array}{l}
V_{\rho}^{(N)}=\sum_{l=0}^{N} \rho^{-l} u_{l}(t, x)=\sum_{l=0}^{N}\left(\rho \alpha(t)^{1+2 \delta}\right)^{-l} g_{l}(t, x), \\
U_{\rho}^{(N)}=e^{i \rho x_{n}} c^{\beta(t, x)} \tilde{\chi}(t) V_{\rho}^{(N)}
\end{array}\right.
$$

We define $\boldsymbol{E}, \widetilde{\boldsymbol{E}}$ as follows.

$$
\begin{aligned}
& \boldsymbol{E}=\left\{b_{\rho}(t, x)=\sum_{j ; \text { finite }} \psi_{j}\left(\alpha(t) \rho^{1 /(1+2 \delta)}\right) h_{j}(t, x)\right. \text { (case (i)) } \\
& \text { or }=\sum_{j ; \text { finite }} \psi_{j}\left(\frac{t}{\widetilde{T}}+\frac{3}{2}\right) h_{j}(t, x) \quad \text { (case (ii)); } \\
&\left.\phi_{j} \in C_{0}^{\infty}([1 / 2,1]), h_{j} \in C_{K}^{\infty}\right\}, \\
& \widetilde{\boldsymbol{E}}=\left\{b_{\rho}(t, x)=\sum_{j ; \text { finite }} \psi_{j}\left(\alpha(t) \rho^{1 /(1+2 \delta)}\right) h_{j}(t, x) \quad\right. \text { (case (i)) } \\
& \text { or }=\sum_{j ; \text { finite }} \psi_{j}\left(\frac{t}{\widetilde{T}}+\frac{3}{2}\right) h_{j}(t, x) \\
&\left.\psi_{j}=\chi \text { or } \in C_{0}^{\infty}([1 / 2,1]), h_{j} \in C_{K}^{\infty}\right\} .
\end{aligned}
$$

Then, we have

$$
\begin{aligned}
\widetilde{P} U_{\rho}^{(N)} & =\rho^{m-1} e^{i \rho x_{n}} e^{\beta(t, x)} L_{\rho}\left(\tilde{\gamma}(t) V_{\rho}^{(N)}(t, x)\right) \\
& =\rho^{m-1} e^{i \rho x_{n}} e^{\beta(t, x)} \sum_{l=0}^{N+1} \rho^{-l} \alpha(t)^{m-1-l(1+2 \delta)-\delta} B_{l}(t, x),
\end{aligned}
$$

where $B_{l} \in \boldsymbol{E}(l=0,1, \cdots, N), B_{l} \in \widetilde{\boldsymbol{E}}(l=N+1, \cdots, N+m-1)$.

We have the estimate (3.1.4) for $\widetilde{P}$ when $U,\left[-T_{0}, T_{1}\right]$ are replaced by $\widetilde{U},\left[-\widetilde{T}_{0}, \widetilde{T}_{1}\right]$. We take $t_{\rho}$ as follows.

$$
\left\{\begin{array}{l}
\text { Case (i) } t_{\rho}=\widetilde{T}_{1}=\widetilde{T}(>0) \\
\text { Case (ii) } t_{\rho}<0, \quad \alpha\left(t_{\rho}\right)=\rho^{-1 /(2(1+2 \delta))}
\end{array}\right.
$$

( $t_{\rho}$ is uniquely determined).

Note that for any $\delta>0$, there exist $g, h \in C^{\infty}\left(\left[-\widetilde{T}_{0}, \widetilde{T}_{1}\right] \times \boldsymbol{R}^{n}\right)$ such that

$$
e^{\beta(t, x)}=\alpha(t)^{\nu(t, x)-\delta} g(t, x)=\alpha(t)^{\nu(t, x)+\delta} \frac{1}{h(t, x)}
$$

and, if $0<\delta<\left|\operatorname{Im} S_{0}(0,0)\right| / 2$, we have, in $\widetilde{U}_{\widetilde{T}_{1}}=\widetilde{U}_{\left[-\widetilde{T}_{0}, \widetilde{T}_{1}\right]}$, 
$\left\{\begin{array}{l}\operatorname{Re} \nu(t, x)-\delta \geqq \operatorname{Re} \nu(0,0)-2 \delta=\operatorname{Im} S_{0}(0,0)-2 \delta>0 \quad \text { (case (i)) } \\ \operatorname{Re} \nu(t, x)+\delta \leqq \operatorname{Re} \nu(0,0)+2 \delta=\operatorname{Im} S_{0}(0,0)+2 \delta<0 \quad \text { (case (ii)). }\end{array}\right.$
Now, we will estimate $U_{\rho}^{(N)}$ and $\widetilde{P} U_{\rho}^{(N)}$ as follows.

Lemma 3. 13. There exist positive constants $\tilde{\delta}$ and $C$ such that for sufficiently large $\rho$ and $N$, there hold the following estimates.

$$
\begin{aligned}
& \left\|U_{\rho}^{(N)}\right\|_{H^{p}\left(\widetilde{U}_{t_{\rho}}\right)} \\
& \geqq \begin{cases}\tilde{\delta} \cdot \rho^{p} & (\text { case }(\mathrm{i})) \\
\tilde{\delta} \cdot \rho^{p-\left(\operatorname{Im} S_{0}(0,0)\right) /(2(1+2 \delta))-2} & (\text { case }(\mathrm{ii})),\end{cases}
\end{aligned}
$$

(3.3.3) $\quad\left\|\widetilde{P} U_{\rho}^{(N)}\right\|_{H^{q}\left(\widetilde{U}_{t_{\rho}}\right)}$

$$
\leqq \begin{cases}C \cdot \rho^{m-1+q-\left(\operatorname{Im} S_{0}(0,0)-3 \delta+m-1\right) /(2(1+2 \delta))} & (\operatorname{case}(\mathrm{i})) \\ C \cdot \rho^{m-1+q} & (\operatorname{case} \text { (ii)) } .\end{cases}
$$

The proof goes on as the proof of Lemma 3.11, so we only point out the different points.

Proof of (3.3.2).

(1) Case (ii), $p \geqq 0$. Note that $\operatorname{Re} \nu(t, x)+\delta \leqq \operatorname{Re} \nu(0,0)+2 \delta$ and $\operatorname{Re} \nu(t, x)-\delta \geqq \operatorname{Re} \nu(0,0)-2 \delta$. By

$$
\tilde{\delta} \alpha(t)^{\operatorname{Re} \nu(0,0)+2 \delta} \leqq e^{\beta(t, x)} \mid \leqq C \alpha(t)^{\operatorname{Re} \nu(0,0)-2 \delta} \quad(\tilde{\delta}>0),
$$

we have

$$
\begin{gathered}
\left\|e^{i \rho x_{n}} e^{\beta(t, x)} \tilde{\chi}(t) u_{0}(t, x)\right\|_{H^{p}}\left(\widetilde{U}_{t_{\rho}}\right) \geqq \tilde{\delta} \rho^{p-(\operatorname{Re} \nu(0,0)+2 \delta) /(2(1+2 \delta))-\delta} \\
\left\|e^{i \rho x_{n}} e^{\beta(t, x)} \tilde{\chi}(t) u_{l}(t, x)\right\|_{H^{p}\left(\widetilde{U}_{t_{\rho}}\right)} \leqq C \rho^{p-(\operatorname{Re} \nu(0,0)+2 \delta) /(2(1+2 \delta))+(l / 2)} \\
(l=1, \cdots, N)
\end{gathered}
$$

So, we get the result by $\operatorname{Re} \nu(0,0)=\operatorname{Im} S_{0}(0,0)$.

(2) Case (ii), $p \leqq 0$. We have, by definition,

$$
\left\|U_{\rho}^{(N)}\right\|_{H^{p}\left(\widetilde{U}_{t \rho}\right)}=\sup _{w \in C_{0}^{\infty}\left(\widetilde{U}_{t \rho}\right)} \frac{\left|\left(U_{\rho}^{(N)}, w\right)_{L^{2}\left(\widetilde{U}_{t \rho}\right)}\right|}{\|w\|_{H^{-p}\left(\widetilde{U}_{t \rho}\right)}} .
$$

Here, we take $w(t, x)=e^{i \rho x_{n}} \varphi(t, x)$, then

$$
\|w\|_{H^{-p}\left(\widetilde{U}_{t \rho}\right)} \leqq \rho^{-p}\|\varphi\|_{H^{-p}\left(\widetilde{U}_{t_{\rho}}\right)},
$$


and so,

$$
\begin{gathered}
\left\|U_{\rho}^{(N)}\right\|_{H^{p}\left(\widetilde{U}_{t \rho}\right)} \geqq \sup _{\varphi \in C_{0}^{\infty}\left(\widetilde{U}_{t \rho}\right)} \frac{\left|\left(e^{\beta(t . x)} \tilde{\chi}(t) V_{\rho}^{(N)}, \varphi\right)_{L^{2}\left(\widetilde{U}_{t \rho}\right)}\right|}{\|\varphi\|_{H^{-p}\left(\widetilde{U}_{t \rho}\right)}} \rho^{p} \\
\geqq \tilde{\delta} \cdot \rho^{p-(\operatorname{Re} \nu(0,0)+2 \delta) /(2(1+2 \delta))-\delta} \quad(\tilde{\delta}>0) .
\end{gathered}
$$

Proof of (3.3.3).

(1) Case (i), $q \geqq 0$. We have only to note that

$$
\begin{aligned}
& U_{\rho}^{(N)}=0 \quad \text { when } \quad \alpha(t) \leqq \frac{1}{2} \rho^{-1 /(2(1+2 \delta))}, \\
& B_{l}=0 \quad(l=0,1, \cdots, N) \quad \text { when } \quad \rho^{-1 /(2(1+28))} \leqq \alpha(t),
\end{aligned}
$$

and

$$
\operatorname{Re} \nu(t, x)-\delta \geqq \operatorname{Re} \nu(0,0)-2 \delta>0 .
$$

(2) When $q \leqq 0$. As in the proof of Lemma 3.11, we can solve

$$
D_{x_{n}}^{|q|} W_{\rho}^{(N)}-\widetilde{P} U_{\rho}^{(N)}=O\left(\rho^{-N-1+m-1}\right),
$$

as

$$
W_{\rho}^{(N)}=\rho^{m-1-|q|} e^{i \rho x_{n}} \sum_{l=0}^{N} \rho^{-l} R_{l}(t, x),
$$

where $R_{l}=\alpha(t)^{\nu(t, x)+m-1-l(1+2 \delta)-2 \delta} \cdot \widetilde{R}_{l}, \widetilde{R}_{l} \in \boldsymbol{E}(l=0,1, \cdots, N)$. And then,

$$
\begin{aligned}
& D_{x_{n}}^{|q|} W_{\rho}^{(N)}-\widetilde{P} U_{\rho}^{(N)}=\rho^{-N-1+m-1} e^{i \rho x_{n}} \\
& \quad \times \alpha(t)^{\nu(t, x)+m-1-(N+m-1)(1+2 \delta)-3 \delta} \sum_{j ; \text { finite }} E_{j}(t, x),
\end{aligned}
$$

where $E_{j} \in \widetilde{\boldsymbol{E}}$. Further, as in the proof of Lemma 3.11,

$$
\left\|D_{x_{n}}^{|q|} W_{\rho}^{(N)}\right\|_{H^{q}\left(\widetilde{U}_{t \rho}\right)} \leqq\left\|W_{\rho}^{(N)}\right\|_{L^{2}\left(\widetilde{U}_{t \rho}\right)} .
$$

So, estimating $\left\|W_{\rho}^{(N)}\right\|_{L^{2}\left(\widetilde{U}_{t_{\rho}}\right)}$ as in the proof of Lemma 3.11, we get the result.

Q.E.D.

From the above lemma, we have

$$
p \leqq m-1+q-\frac{1}{2(1+2 \delta)}\left(\operatorname{Im} S_{0}(0,0)-3 \delta+m-1\right) \quad(\text { case (i)) }
$$




$$
p-\frac{\operatorname{Im} S_{0}(0,0)}{2(1+2 \delta)}-2 \delta \leqq m-1+q \quad \text { (case (ii)) }
$$

So, by letting $\delta \rightarrow+0$, we have

$$
\begin{aligned}
& \pm \operatorname{Im} S_{0}(0,0) \leqq 2(q+m-1-p) \\
& \quad(+ \text { in the case (i), }- \text { in the case (ii) }) .
\end{aligned}
$$

Q.E.D.

\section{Appendix I. Proof of (2.1.3)}

The case $d=1$ follows from the result of Ivrii [8]. But we will give a simple proof.

(1) When $P=P_{2}=D_{t}^{2}-t D_{x}^{2}, d=1, p=0$. First, we fix $t_{0} \in(0, T)$, and for $u \in \mathscr{S}\left(\boldsymbol{R}^{2}\right)$ we put

$$
w(t, x)=\int_{t}^{t_{0}} u\left(t^{\prime}, x\right) d t^{\prime} .
$$

Then,

$$
w\left(t_{0}, x\right)=0, \quad \partial_{t} w(t, x)=-u(t, x) .
$$

Now, we have

$$
\begin{aligned}
2 \operatorname{Re} & \int_{0}^{t_{0}} \int P_{2} u \cdot \overline{w_{x x}} d x d t \\
= & 2 \operatorname{Re} \int_{0}^{t_{0}} \int u_{t t x} \overline{w_{x}} d x d t-\int_{0}^{t_{0}} \int t \partial_{t}\left(\left|w_{x x}\right|^{2}\right) d x d t \\
= & 2 \operatorname{Re}\left[\int u_{t x} \overline{w_{x}} d x\right]_{0}^{t_{0}}+2 \operatorname{Re} \int_{0}^{t_{0}} \int u_{t x} \overline{u_{x}} d x d t-\left[t \int\left|w_{x x}\right|^{2} d x\right]_{0}^{t_{0}} \\
& +\int_{0}^{t_{0}} \int\left|w_{x x}\right|^{2} d x d t=-2 \operatorname{Re} \int u_{t x}(0, x) \overline{w_{x}(0, x)} d x \\
& +\int_{0}^{t_{0}} \int \partial_{t}\left(\left|u_{x}\right|^{2}\right) d x d t+\int_{0}^{t_{0}} \int\left|w_{x x}\right|^{2} d x d t \\
= & \int\left|u_{x}\left(t_{0}, x\right)\right|^{2} d x-\int\left|u_{x}(0, x)\right|^{2} d x \\
& +\int_{0}^{t_{0}} \int\left|w_{x x}\right|^{2} d x d t-2 \operatorname{Re} \int u_{t x}(0, x) \overline{w_{x}(0, x)} d x .
\end{aligned}
$$

Here, 
236

TAKESHI MANDAI

$$
w_{x}(0, x)=\int_{0}^{t_{0}} u_{x}(t, x) d t
$$

so,

$$
\int\left|w_{x}(0, x)\right|^{2} d x \leqq T \int_{0}^{t_{0}} \int\left|u_{x}(t, x)\right|^{2} d x d t
$$

Thus, we have

$$
\begin{aligned}
& \int\left|u_{x}\left(t_{0}, x\right)\right|^{2} d x+\int_{0}^{t_{0}} \int\left|w_{x x}\right|^{2} d x d t \\
& \leqq \int_{0}^{t_{0}} \int\left|P_{2} u\right|^{2} d x d t+\int_{0}^{t_{0}} \int\left|w_{x x}\right|^{2} d x d t+\int\left|u_{x}(0, x)\right|^{2} d x \\
& \quad+\int\left|u_{t x}(0, x)\right|^{2} d x+T \int_{0}^{t_{0}} \int\left|u_{x}(t, x)\right|^{2} d x d t .
\end{aligned}
$$

By Gronwall's lemma, we have

(A. 1)

$$
\begin{aligned}
& \int\left|u_{x}\left(t_{0}, x\right)\right|^{2} d x \leqq C\left\{\int_{0}^{t_{0}} \int\left|P_{2} u\right|^{2} d x d t\right. \\
& \left.+\int\left|u_{x}(0, x)\right|^{2} d x+\int\left|u_{t x}(0, x)\right|^{2} d x\right\} \text { for } t_{0} \in(0, T) .
\end{aligned}
$$

Next,

$$
\begin{aligned}
-2 \operatorname{Re} \int_{0}^{t} \int P_{2} u \cdot \overline{u_{t}} d x d t^{\prime} \\
=2 \operatorname{Re} \int_{0}^{t} \int u_{t t} \overline{u_{t}} d x d t^{\prime}-2 \operatorname{Re} \int_{0}^{t} \int t^{\prime} u_{x x} \overline{u_{t}} d x d t^{\prime} \\
=\int_{0}^{t} \int \partial_{t}\left(\left|u_{t}\right|^{2}\right) d x d t^{\prime}+2 \operatorname{Re} \int_{0}^{t} \int t^{\prime} u_{x} \overline{u_{x t}} d x d t^{\prime} \\
=\int\left|u_{t}(t, x)\right|^{2} d x-\int\left|u_{t}(0, x)\right|^{2} d x+\int_{0}^{t} \int t^{\prime} \partial_{t}\left(\left|u_{x}\right|^{2}\right) d x d t^{\prime} \\
=\int\left|u_{t}(t, x)\right|^{2} d x-\int\left|u_{t}(0, x)\right|^{2} d x+\left[t^{\prime} \int\left|u_{x}\right|^{2} d x\right]_{0}^{t} \\
\quad-\int_{0}^{t} \int\left|u_{x}\right|^{2} d x d t^{\prime}=\int\left|u_{t}(t, x)\right|^{2} d x+t \int\left|u_{x}(t, x)\right|^{2} d x \\
\quad-\int\left|u_{t}(0, x)\right|^{2} d x-\int_{0}^{t} \int\left|u_{x}\right|^{2} d x d t^{\prime} .
\end{aligned}
$$

Thus, 


$$
\begin{aligned}
& \int\left|u_{t}(t, x)\right|^{2} d x \leqq \int_{0}^{t} \int\left|P_{2} u\right|^{2} d x d t^{\prime}+\int_{0}^{t} \int\left|u_{t}\left(t^{\prime}, x\right)\right|^{2} d x d t^{\prime} \\
& +\int_{0}^{t} \int\left|u_{x}\right|^{2} d x d t^{\prime}+\int\left|u_{t}(0, x)\right|^{2} d x \leqq C \int_{0}^{t} \int\left|P_{2} u\right|^{2} d x d t^{\prime} \\
& +\int_{0}^{t} \int\left|u_{t}\left(t^{\prime}, x\right)\right|^{2} d x d t^{\prime}+C\left\{\int\left|u_{x}(0, x)\right|^{2} d x\right. \\
& \left.+\int\left|u_{t x}(0, x)\right|^{2} d x\right\}+\int\left|u_{t}(0, x)\right|^{2} d x .
\end{aligned}
$$

By Gronwall's lemma, we have

$$
\begin{gathered}
\text { (A. 2) } \quad \int\left|u_{t}(t, x)\right|^{2} d x \leqq C\left\{\int_{0}^{t} \int\left|P_{2} u\right|^{2} d x d t^{\prime}+\int\left|u_{t}(0, x)\right|^{2} d x\right. \\
\left.+\int\left|u_{x}(0, x)\right|^{2} d x+\int\left|u_{t x}(0, x)\right|^{2} d x\right\} .
\end{gathered}
$$

Lastly, we have

(A. 3) $\int|u(t, x)|^{2} d x \leqq C\left(\int_{0}^{t} \int\left|u_{t}\left(t^{\prime}, x\right)\right|^{2} d x d t^{\prime}+\int|u(0, x)|^{2} d x\right)$.

From (A. 1), (A. 2), (A. 3), we have

$$
\begin{gathered}
\sum_{j=0}^{1}\left\|D_{t}^{j} u(t, \cdot)\right\|_{1-j}^{2} \leqq C\left(\int_{0}^{t}\left\|P_{2} u\left(t^{\prime}, \cdot\right)\right\|_{0}^{2} d t^{\prime}+\sum_{j=0}^{1}\left\|D_{t}^{j} u(0, \cdot)\right\|_{1}^{2}\right) \\
\text { for } 0 \leqq t \leqq T, u \in \mathscr{S}\left(\mathbb{R}^{2}\right) .
\end{gathered}
$$

By substituting $\left(1+D_{x}^{2}\right)^{s / 2} u$ for $u$, we have the result.

(2) When $P=P_{2}, d=1, p>0$. We prove by induction on $p$. By induction hypothesis, we have

$$
\begin{aligned}
\sum_{j=0}^{p+1} \| & D_{t}^{j} u(t, \cdot) \|_{s+1-j}^{2} \\
& \leqq C\left(\sum_{j=0}^{p} \int_{0}^{t}\left\|D_{t}^{j} P_{2} u\left(t^{\prime}, \cdot\right)\right\|_{s-j}^{2} d t^{\prime}+\sum_{j=0}^{p+1}\left\|D_{t}^{j} u(0, \cdot)\right\|_{s+2-j}^{2}\right) .
\end{aligned}
$$

Here, by $P_{2} D_{t}=D_{t} P_{2}-i D_{x}^{2}$, we have (by substituting $D_{t} u$ for $u$ )

$$
\begin{aligned}
\sum_{j=0}^{p+1} \| & D_{t}^{j+1} u(t, \cdot) \|_{s+1-j}^{2} \leqq C\left\{\sum_{j=0}^{p} \int_{0}^{t}\left\|D_{t}^{j+1} P_{2} u\left(t^{\prime}, \cdot\right)\right\|_{s-j}^{2} d t^{\prime}\right. \\
& \left.+\sum_{j=0}^{p} \int_{0}^{t}\left\|D_{t}^{j} u\left(t^{\prime}, \cdot\right)\right\|_{s+2-j}^{2} d t^{\prime}+\sum_{j=0}^{p+1}\left\|D_{t}^{j+1} u(0, \cdot)\right\|_{s+2-j}^{2}\right\} .
\end{aligned}
$$


Therefore, we have

$$
\begin{aligned}
\sum_{j=0}^{p+2} \| & D_{t}^{j} u(t, \cdot) \|_{s+2-j}^{2} \leqq C\left\{\sum_{j=0}^{p+1} \int_{0}^{t}\left\|D_{t}^{j} P_{2} u\left(t^{\prime}, \cdot\right)\right\|_{s+1-j}^{2} d t^{\prime}\right. \\
& \left.+\sum_{j=0}^{p} \int_{0}^{t}\left\|D_{t}^{j} u(t, \cdot)\right\|_{s+2-j}^{2} d t^{\prime}+\sum_{j=0}^{p+2}\left\|D_{t}^{j} u(0, \cdot)\right\|_{s+3-j}^{2}\right\}
\end{aligned}
$$

Again by Gronwall's lemma, we have the case $p+1$.

(3) General case. For $Q=a(t, x) D_{t}+b(t, x) D_{x}+c(t, x) \quad(a, b, c$ $\left.\in \mathscr{B}^{\infty}([0, T] \times \boldsymbol{R})\right)$, we have

$$
\begin{aligned}
\sum_{j=0}^{p} \| & D_{t}^{j} Q u(t, \cdot) \|_{s-j}^{2} \leqq C\left\{\sum_{j=0}^{p} \int_{0}^{t}\left\|D_{t}^{j} P_{2} u\left(t^{\prime}, \cdot\right)\right\|_{s-j}^{2} d t^{\prime}\right. \\
& \left.+\sum_{j=0}^{p+1}\left\|D_{t}^{j} u(0, \cdot)\right\|_{s+2-j}^{2}\right\} .
\end{aligned}
$$

So, as in Section 1.2, we get the result for $P=P_{2}+Q, d=1$. Successive uses of this result for $d=1$ allows us to get the result for $d>1$. (See the proof of Theorem 1.9.)

Q.E.D.

\section{Appendix II}

In this appendix, $\alpha(t)$ and $\left[-T_{0}, T_{1}\right]$ are the same as in Section 3. 1 .

Lemma. For any $f \in C^{\infty}\left(\left[-T_{0}, T_{1}\right] \times \boldsymbol{R}^{n}\right)$ and any positive number $\nu$, there exists $F \in C^{\infty}\left(\left[-T_{0}, T_{1}\right] \times \boldsymbol{R}^{n}\right)$ such that

$$
\begin{gathered}
\int_{0}^{t} \alpha(u)^{\nu} f(u, x) d u=\alpha(t)^{\nu} t^{\omega+1} F(t, x) \\
\text { for } \quad(t, x) \in\left[-T_{0}, T_{1}\right] \times \boldsymbol{R}^{n} .
\end{gathered}
$$

Proof. By

$$
\alpha^{\prime}(t)=b(t)|t|^{-\omega-1} \alpha(t), b(t) \neq 0 \quad \text { for } t \in\left[-T_{0}, T_{1}\right],
$$

we have

$$
\begin{aligned}
& \int_{0}^{t} \alpha(u)^{\nu} f(u, x) d u=\left[\alpha(u)^{\nu} \cdot \frac{|u|^{\omega+1}}{\nu b(u)} f(u, x)\right]_{0}^{t} \\
& \quad-\int_{0}^{t} \alpha(u)^{\nu} u^{\omega} f_{1}(u, x) d u \quad \text { for } f_{1} \in C^{\infty}\left(\left[-T_{0}, T_{1}\right] \times \boldsymbol{R}^{n}\right) .
\end{aligned}
$$


Thus, by induction, for any positive integer $N$, there exist $F_{N}, f_{N} \in$ $C^{\infty}\left(\left[-T_{0}, T_{1}\right] \times \boldsymbol{R}^{n}\right)$ such that

$$
\int_{0}^{t} \alpha(u)^{\nu} f(u, x) d u=\alpha(t)^{\nu} t^{\omega+1} F_{N}(t, x)+\int_{0}^{t} \alpha(u)^{\nu} u^{N \omega} f_{N}(u, x) d u .
$$

We put

$$
G_{N}(t, x)=\frac{1}{\alpha(t)^{\nu} t^{\omega+1}} \int_{0}^{t} \alpha(u)^{\nu} u^{N \omega} f_{N}(u, x) d u .
$$

We have only to prove that for any positive integer $M$, there exists positive integer $N$ such that $G_{N} \in C^{M}\left(\left[-T_{0}, T_{1}\right] \times \mathbb{R}^{n}\right)$.

First,

$$
G_{N}(t, x)=\int_{0}^{t}(\alpha(u) / \alpha(t))^{\nu}(u / t)^{\omega+1} u^{N \omega-\omega-1} f_{N}(u, x) d u,
$$

and,

$$
|\alpha(u) / \alpha(t)| \leqq 1,|u / t| \leqq 1 \text { for } 0 \leqq|u| \leqq|t| .
$$

So, we have

$$
G_{N}(t, x)=O\left(t^{N \omega-\omega}\right) \quad(t \rightarrow 0)
$$

Next,

$$
\partial_{t} G_{N}=-\left\{\nu b(t)|t|^{--\omega-1}+(\omega+1) t^{-1}\right\} G_{N}+t^{N \omega-\omega-1} f_{N}(t, x) .
$$

By induction, there exist $g_{l}, h_{l} \in C^{\infty}\left(\left[-T_{0}, T_{1}\right] \times \mathbb{R}^{n}\right)(l=0,1, \cdots)$ such that

$$
\partial_{t}^{l} G_{N}=\frac{g_{l}(t, x)}{t^{l(\omega+1)}} G_{N}(t, x)+t^{N \omega-l(\omega+1)} h_{l}(t, x) \quad(l=0,1, \cdots) .
$$

So, we have

$$
\left(\partial_{t}^{l} G_{N}\right)(t, x)=O\left(t^{N \omega-\omega-l(\omega+1)}\right) \quad(t \rightarrow 0) .
$$

Thus, if $N \omega-\omega-M(\omega+1)>0$, we have $G_{N} \in C^{M}\left(\left[-T_{0}, T_{1}\right] \times \mathbb{R}^{n}\right)$,

Q.E.D.

\section{References}

[1] Alinhac, S., Paramétrix et propagation des singularités pour un problème de Cauchy à multiplicité variable, Asterisque, 34-35 (1976), 3-26.

[2] - Solution explicite du problème de Cauchy pour des opérateurs effectivement hyperboliques, Duke Math. J., 45 (1978), 225-258.

[3] Chazarain, J., Opérateurs hyperboliques à caracteristiques de multiplicité constante, Ann. Inst. Fourier, 24 (1974), 173-202.

[4] Chi Min-You, On the Cauchy problem for a class of hyperbolic equations with data 
given on the degenerate parabolic line, Acta Math. Sinica, 8 (1958), 521-529. (Chinese Math. (1967), 246-254.)

[5] Hörmander, L., The Cauchy problem for differential equations with double characteristics, J. Analyse Math., 32 (1977), 118-196.

[6] Igari, K., Cauchy problem for weakly hyperbolic equations of second order, $J$. Math. Kyoto Univ., 20 (1980), 43-55.

[7] Ivrii, V. Y. and Petkov, V. M., Necessary conditions for the Cauchy problem for non-strictly hyperbolic equations to be well-posed, Uspehi Mat. Nauk, 29 (1974), 3-70. (Russian Math. Surveys, 29 (1974), 1-70.)

[8] Ivrii, V. Y., Sufficient conditions for regular and completely regular hyperbolicity, Trudy Moscov Obsc., 33 (1976), 3-66.(Transaction of Moscow Math. Soc., 33 (1978), 1-65.)

[9] Kitagawa, K. and Sadamatsu, T., Sur une condition suffisante pour que le problème de Cauchy faiblement hyperbolique soit bien posé-Cas de multiplicité de characteristiques au plus triple, J. Math. Kyoto Univ., 17 (1977), 465-499.

[10] Komatsu, H., Linear hyperbolic equations with Gevrey coefficients, J. Math. Pures Appl., 59 (1980), 145-185.

[11] Kumano-go, H., Fundamental solution for a hyperbolic system with diagonal principal part, Comm. in Partial Differential Equations, 4 (1979), 959-1015.

[12] Menikoff, A., The Cauchy problem for weakly hyperbolic equations, Amer. J. Math., 97 (1975), 548-558.

[13] Nakamura, G. and Uryu, H., Parametrix of certain weakly hyperbolic operators, Comm. in Partial Differential Equations, 5 (1980), 837-896.

[14] Nishitani, T., Some remarks on the Cauchy problem for weakly hyperbolic equations, J. Math. Kyoto Univ., 17 (1977), 245-258.

[15] - On the Cauchy problem for weakly hyperbolic equations, Comm. in Partial Differential Equations, 3 (1978), 319-333.

[16] Ohya, Y., Le problème de Cauchy à caracteristique multiples, Ann. Scuola Norm. Sup. Pisa, 4 (1977), 757-805.

[17] Olejnik, O. A., On the Cauchy problem for weakly hyperbolic equations, Comm. Pure Appl. Math., 23 (1970), 569-586.

[18] Peyser, G., Energy inequalities for hyperbolic equations in several variables with multiple characteristics and constant coefficients, Trans. Amer. Math. Soc, 108 (1963), 478-490.

[19] Svensson, S. L., Necessary and sufficient conditions for the hyperbolicity of polynomials with hyperbolic principal part, Ark. Mat., 8 (1969), 145-162.

[20] Tahara, H., Singular hyperbolic systems, III. On the Cauchy problem for Fuchsian hyperbolic partial differential equations, J. Fac. Sci. Univ. Tokyo, 27 (1980), 465507.

[21] Tarama, S., Un exemple dans le problème de Cauchy pour les équations faiblement hyperboliques, Publ. RIMS. Kyoto Univ., 15 (1979), 455-468.

[22] Uryu, H., The Cauchy problem for weakly hyperbolic equations, Comm. in Partial Differential Equations, 5 (1980), 23-40.

[23] Wakabayashi, S., The Cauchy problem for operators with constant coefficient hyperbolic principal part and propagation of singularities, Japan. J. Math., 6 (1980), 179-228.

[24] Yoshikawa, A., Construction of a parametrix for the Cauchy problem of some weakly hyperbolic equations, I, II, III, Hokkaido Math. J., 6 (1977), 313-344, 7 (1978), 1-26, 127-141.

[25] - Parametrices for a class of effectively hyperbolic operators, Comm. in Partial Differential Equations, 5 (1980), 1073-1151. 